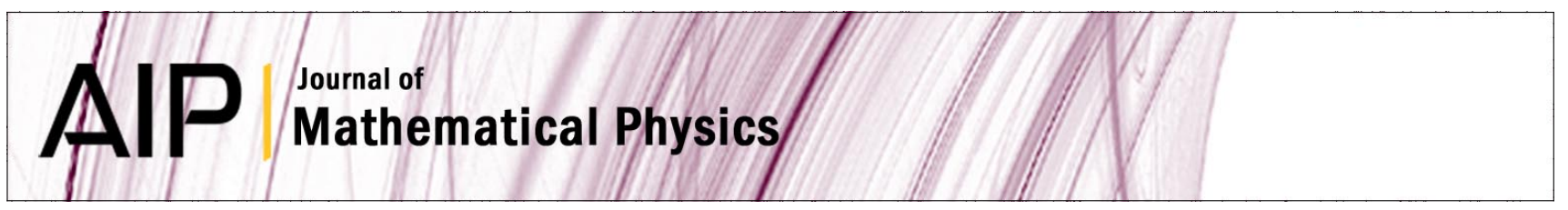

\title{
Anisotropic Einstein-aether cosmological models
}

B. Alhulaimi, A. Coley, and P. Sandin

Citation: J. Math. Phys. 54, 042503 (2013); doi: 10.1063/1.4802246

View online: http://dx.doi.org/10.1063/1.4802246

View Table of Contents: http://jmp.aip.org/resource/1/JMAPAQ/v54/i4

Published by the American Institute of Physics.

\section{Additional information on J. Math. Phys.}

Journal Homepage: http://jmp.aip.org/

Journal Information: http://jmp.aip.org/about/about_the_journal

Top downloads: http://jmp.aip.org/features/most_downloaded

Information for Authors: http://jmp.aip.org/authors

\section{ADVERTISEMENT}

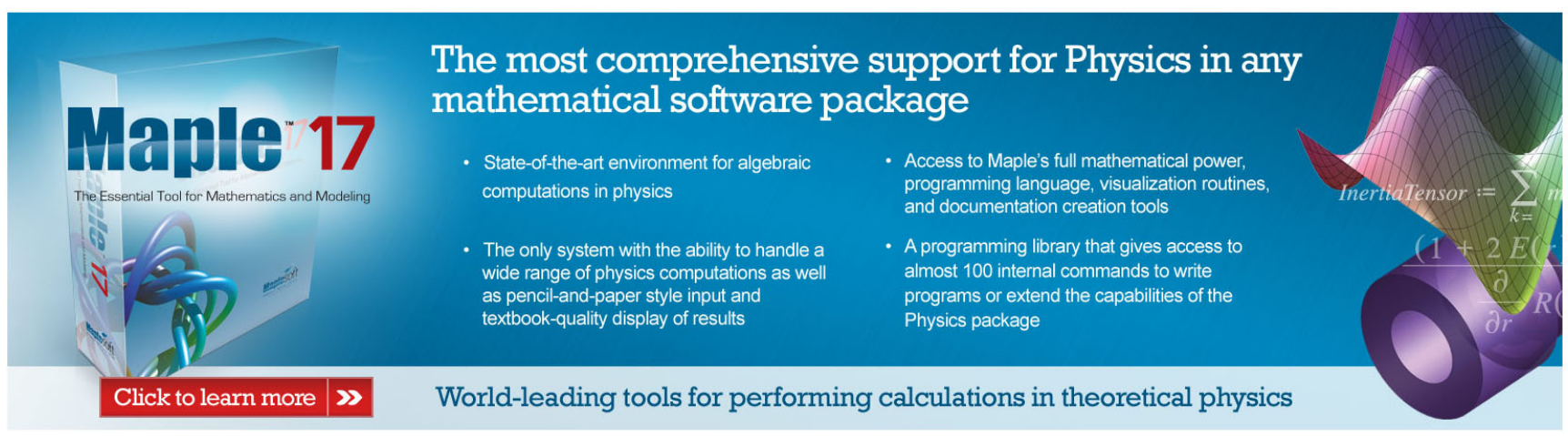




\title{
Anisotropic Einstein-aether cosmological models
}

\author{
B. Alhulaimi, ${ }^{1, \text { a) }}$ A. Coley, ${ }^{1, b)}$ and P. Sandin ${ }^{1,2, c)}$ \\ ${ }^{1}$ Department of Mathematics, Dalhousie University, B3H $3 J 5$ Halifax, Canada \\ ${ }^{2}$ Max-Planck-Institut für Gravitationsphysik (Albert-Einstein-Institut), Am Mühlenberg 1, \\ D-14476 Potsdam, Germany
}

(Received 21 November 2012; accepted 2 April 2013; published online 30 April 2013)

\begin{abstract}
We investigate a class of spatially anisotropic cosmological models in Einsteinaether theory with a scalar field in which the self-interaction potential depends on the timelike aether vector field through the expansion and shear scalars. We derive the evolution equations in terms of expansion-normalized variables, which reduce to a dynamical system. We study the local stability of the equilibrium points of the dynamical system corresponding to physically realistic solutions, and find that there are always ranges of values of the parameters of the models for which there exists an inflationary attractor. ( 2013 AIP Publishing LLC. [http://dx.doi.org/10.1063/1.4802246]
\end{abstract}

\section{INTRODUCTION}

There are various models of early universe cosmology which incorporate a violation of Lorentz invariance, which include Einstein-aether theory ${ }^{1,2}$ and the IR limit of (extended) Horava gravity. ${ }^{3}$ When the phenomenology of theories with a preferred frame is studied, it is generally assumed that this frame coincides, at least roughly, with the cosmological rest frame defined by the Hubble expansion of the universe. It has recently been shown, ${ }^{4}$ based on a Bayesian analysis and assuming nothing other than the existence of a suitably averaged linear Hubble law, that the standard Cosmic Microwave Background (CMB) rest frame is not the one in which the Hubble flow is most uniform (questioning whether the standard treatment of the cosmic rest frame in which the variance in the Hubble flow can be reduced to a boost at a point is correct).

Einstein-aether theory consists of general relativity (GR) coupled, at second derivative order, to a dynamical timelike unit vector field, the aether. In Horava gravity, the aether vector is assumed to be hypersurface-orthogonal; hence every hypersurface-orthogonal Einstein-aether solution is a Horava solution (most of the solutions studied). In this effective field theory approach, the aether vector field $u_{a}$ and the metric tensor $g_{a b}$ together determine the local spacetime structure.

The inflationary paradigm ${ }^{5}$ provides one of the simplest ways to describe various aspects of the physics of the early universe in standard cosmology. However, despite its successes some of the fundamental questions still remain to be answered in this paradigm. We shall discuss the late time dynamics of Einstein aether cosmological models. In particular, we explore the impact of Lorentz violation on the inflationary scenario. ${ }^{1,2,6-8}$

Cosmological models in aether (Lorentz-violating) theories of gravity are currently of interest. A systematic construction of an Einstein-aether gravity theory with a Lorentz violating dynamical field that preserves locality and covariance in the presence of an additional "aether" vector field has been presented (the field equations (FE) are given therein). ${ }^{1,9,10}$

In an isotropic and spatially homogeneous Friedmann universe with expansion scale factor $a(t)$ and comoving proper time $t$, the aether field will be aligned with the cosmic frame and is related to the expansion rate of the universe. The Einstein FE are generalised by the contribution of an additional stress tensor for the aether field. If the universe contains a single self-interacting scalar field $\phi$ (e.g.,

\footnotetext{
a)Electronic mail: Bassemah@mathstat.dal.ca

b) Electronic mail: aac@ mathstat.dal.ca

c)Electronic mail: patrik.sandin@aei.mpg.de
} 
a scalar inflation which would dominate in any inflationary epoch), with a self-interaction potential $V, V$ can now be a function of $\phi$ and the expansion rate and even the shear. ${ }^{1}$

\section{A. Exponential potentials}

Exponential potentials $V_{0} e^{-\lambda \phi}$ arise naturally in various higher dimensional frameworks, such as in Kaluza-Klein theories and supergravity. ${ }^{11}$ Scalar fields with exponential potentials in GR do not yield exponential inflation as with (for example) the harmonic potential. ${ }^{5}$ However, they do lead to a power-law inflation provided the potential is not too steep. The limitation of steep potentials can be alleviated using multiple fields, which can cooperate via Hubble damping to yield assisted inflation. ${ }^{12}$ In addition, for sufficiently flat potentials, exponential potentials yield a scaling solution which is a late time attractor. ${ }^{13,14}$

The dynamical properties of the positive exponential potentials leading to inflation in the Friedmann-Robertson-Walker (FRW) model have been widely studied ${ }^{15-17}$ (also see Appendix A). The classical solution for the scale factor can be written as a power law, $a \propto t^{n}$, with $n=2 / \lambda^{2}$; in order to have an inflationary phase with the exponential potential, one requires the steepness parameter $\lambda$ to satisfy $\lambda^{2}<2$. If the potential is steep, then it does not support inflation. The effective dynamics with an exponential potential have been more widely studied. ${ }^{13,14}$ The equilibrium points corresponding to sources consist of a subset of the (non-oscillatory) Jacobs anisotropic Bianchi I non-vacuum (massless scalar field) solutions. ${ }^{14}$

Negative exponential potentials also lead to a rich physics, such as in the Ekpyrotic scenario. ${ }^{18}$

\section{ANISOTROPIC MODELS}

We study a class of spatially homogeneous, anisotropic cosmological models in Einstein-aether theory. Carruthers and Jacobson ${ }^{6}$ and Kanno and Soda ${ }^{7}$ examined Bianchi type I anisotropic solutions in the presence of a positive cosmological constant, with three orthogonal principal directions of expansion, and with the aether tilted in one of the principal directions. They showed that, to linear order in the anisotropy, the system relaxes exponentially to the isotropic, de Sitter solution.

In an anisotropic Einstein-aether model there will be additional terms in the FE:

- The effects on the geometry from the anisotropy (and curvature) of the actual 1-parameter subclass of Bianchi $V I_{h}$ spatially homogeneous models (with an isotropic curvature; see Eqs. (4)-(5)) considered below.

- The energy momentum tensor of the scalar field, due to the possible dependence of the selfinteraction potential $V$ on the Lorentz violating vector field (see Eqs. (14)-(15) below). ${ }^{9}$

- The Einstein FE are generalised by the contribution of an additional stress tensor, $S_{a b}$, for the aether field which depends on the dimensionless parameters of the aether model (e.g., "the $c_{i}$ "). This has the effect of renormalizing some of the parameters in the model (e.g., the gravitational constant $G$, where we choose units in which $8 \pi G=1$; effectively we set $c_{1}+3 c_{2}+c_{3}=0$ so that the remaining parameters in the model can be characterized by the constants $c^{2}$ and $d$ - see below). ${ }^{7}$

- In anisotropic models, there may be a tilt between the preferred direction of the aether and that of the anistropy (in an isotropic and spatially homogeneous Friedmann universe the aether field is aligned with the cosmic frame). This adds additional terms to the aether stress tensor $S_{a b}$, which can be characterized by a hyperbolic tilt angle, $\alpha(t)$, measuring the boost of the aether relative to the rest frame of the homogeneous spatial sections. ${ }^{6,7}$

In this paper, we are interested in the qualitative features of cosmological models in Einstein aether theory (and in particular in the presence of curvature and shear).

\section{A. The potential}

If the universe contains a single self-interacting scalar field $\phi$ (e.g., a scalar inflation which would dominate in any inflationary epoch), the self-interaction potential $V$ is a function of $\phi$ but 
can now also be a function of the timelike vector field $u_{a}$ (the aether field) ${ }^{1} V=V(\phi, \theta, \sigma)$, where $\theta=3 H=3 \dot{a} / a$ is the expansion rate and $\sigma$ is the shear scalar, defined by $\sigma^{2} \equiv \frac{1}{2} \sigma^{a b} \sigma_{a b}$. The modified stress tensor $T_{a b}^{\phi}$ can be written in terms of an effective fluid with density $\rho_{\phi}$ and pressure $p_{\phi}$ (see below).

In general, we consider a scale invariant potential of the form (cf. Ref. 9),

$$
V(\theta, \phi, \sigma)=V_{0} \exp [-\lambda \phi]+\sum_{r, s} a_{r, s} \theta^{r} \sigma^{s} \exp [(r+s-2) \lambda \phi / 2]
$$

where $V_{0}, \lambda$ ( $k \equiv-\lambda$ in Ref. 19 and Appendix A) and $\left\{a_{r, s}\right\}$ are constants. The constant $V_{0}$ is assumed to be positive. Negative constants $a_{r, s}$ are permitted; however, it might be demanded that the potential $V(\theta, \phi, \sigma)$ is positive definite.

In particular, we shall study the particular potential

$$
V(\theta, \phi)=V_{0} e^{-\lambda \phi}+a \sqrt{V_{0}} \theta e^{-\frac{1}{2} \lambda \phi}+b \sqrt{V_{0}} \sigma e^{-\frac{1}{2} \lambda \phi},
$$

where, for convenience, we have renormalized the constants, dropped the indices on the constants and renamed the second constant $b$. We could easily consider additional terms with $r=2, s=0$ and $r=0, s=2$, but these terms are expected to simply renormalize the arbitrary parameters in the resulting model.

We could also study a potential with a single generic term (with arbitrary values for $r$, $s$, and non-zero constant $\left.a_{r, s}\right) .^{20}$

\section{B. The model}

The evolution equations follow from the FE derived from the Einstein aether action. ${ }^{1,2}$ The energy-momentum conservation law or Klein-Gordon equation is

$$
\ddot{\phi}+\theta \dot{\phi}+V_{\phi}=0 \text {. }
$$

The generalized Friedmann equation is

$$
\theta^{2}=3 c^{2} \sigma^{2}+9 d\left(\dot{\alpha}+\frac{1}{3} \alpha \theta\right)^{2}+3 \rho_{\phi}-\frac{3}{2} P
$$

where $P$ is the scalar curvature of the homogeneous hypersurfaces, which is always negative in the models discussed here (where we also neglect normal matter). The Raychaudhuri equation governing the evolution of the expansion is given by

$$
\dot{\theta}=-\frac{1}{3} \theta^{2}-2 c^{2} \sigma^{2}+d\left(\alpha^{2}\left(\dot{\theta}+\frac{1}{3} \theta^{2}\right)-3 \dot{\alpha}^{2}+\alpha \dot{\alpha} \theta+3 \alpha \ddot{\alpha}\right)-\frac{1}{2}\left(\rho_{\phi}+3 p_{\phi}\right) .
$$

Following Ref. 21 (also see Appendix A), we consider a 1-parameter $(m \equiv h-1)$ class of anisotropic cosmological models with metric (A10), which includes Bianchi types III $(m=0), \mathrm{V}$ $(m=1), \mathrm{VI}_{0}(m=-1)$, and $\mathrm{VI}_{h}$ (all other $m$ ). The expansion scalar, which determines the volume behavior of the fluid, is given by (A11) and the shear scalar is given by (A13). In the case under consideration here, there is no rotation and no acceleration; i.e.,

$$
u_{a ; b}=\frac{1}{3} \theta h_{a b}+\sigma_{a b}
$$

where $h_{a b} \equiv g_{a b}+u_{a} u_{b}$.

Thus, in our model, in the generalized Friedmann equation $-\frac{3}{2} P=\frac{3}{a^{2}} N$, where $N \equiv m^{2}+m$ $+1 \geq 3 / 4>0$. Using the generalized Friedmann equation, the evolution equation for the shear is

$$
c^{2} \dot{\sigma}=-c^{2} \sigma \theta+\frac{M}{3 \sqrt{3}}\left(\theta^{2}-3 c^{2} \sigma^{2}-9 d\left(\dot{\alpha}+\frac{1}{3} \alpha \theta\right)^{2}-3 \rho_{\phi}\right),
$$

where $M \equiv \frac{1-m}{\sqrt{m^{2}+m+1}}$. The constants $M$ and $N$ are not independent but are related (via $m$ ); formally, we can recover the Bianchi type I case by taking $M=N=0$. 
Finally, the evolution equation for the tilt is

$$
\ddot{\alpha}+\theta \dot{\alpha}+\frac{1}{9} \alpha\left(3 \dot{\theta}+2 \theta^{2}\right)=0 .
$$

\section{The future evolution of tilt}

The evolution equation (the "master" equation in Ref. 7) for the tilt (in the small anisotropy and small tilt limit; i.e., using the Raychaudhuri equation and keeping $\alpha, \sigma$ to first order only) is given by

$$
\ddot{\alpha}+\theta \dot{\alpha}-\frac{1}{9} \alpha\left[\theta^{2}+\frac{27}{2}\left(\rho_{\phi}+3 p_{\phi}\right)\right]=0
$$

From this equation it was shown that the tilt decays $(\alpha \rightarrow 0)$ to the future (in the Bianchi type I models) as is expected. ${ }^{7}$ (Note that the possibility that the dimensionless parameters can depend on the cosmological time via the scalar field (i.e., $\left.c_{i}=c_{i}(\phi)\right)$ was also considered by Ref. 7 , and it was found that inflationary solutions can be obtained even in the absence of a scalar field potential, as in scalar-tensor theories of gravity.) The dynamics of a tilted aether in a Bianchi I cosmological model without the assumption of a small tilt was studied in Ref. 6, and it was found that when the initial hyperbolic tilt angle $\alpha$ (and its time derivative) is sufficiently small, then $\alpha \rightarrow 0$ at late times (consistent with the linearized stability analysis in Ref. 7), but in general (for larger tilt angles) there can be runaway (or singular) behaviour in the anisotropy. It is known that the tilt decays to the future in general tilted perfect fluid Bianchi cosmological models (except in the degenerate case in which the tilt becomes extreme; but this case must be treated separately here). ${ }^{22}$

\section{Past evolution}

We are also interested in the past evolution of these models. Let us discuss this briefly. Since we expect the spatial curvature not to be dynamically important to the past, as a first step we can study the Bianchi type I models. We need to study the tilt carefully, since the tilt may become extreme to the past (and we may need to parameterize the tilt differently).

In the case of a stiff perfect fluid (or massless scalar field) in GR, the solutions are nonoscillatory to the past, and asymptote towards a one parameter family of sources that correspond to Jacobs anisotropic Bianchi type I non-vacuum solutions. ${ }^{13}$ We shall later find that analogues of the Jacobs anisotropic solutions are sources of the anisotropic Einstein aether models. Let us simply ask whether the tilt could change the qualitative picture. The normalized equations for the tilt angle $\alpha$ are

$$
\alpha^{\prime}=3 \Pi, \Pi^{\prime}=(q-2) \Pi+(q-1) \alpha,
$$

where $\Pi \equiv \dot{\alpha} / \theta$. The equilibrium points with $q=2$ (the analogues of the Jacobs solutions) are not compatible with $\alpha$ non-zero, which implies that there are no sources with non-zero tilt. A local stability analysis of these equilibrium points (with zero tilt) then shows that all of the non-zero eigenvalues (the equilibrium points are non-isolated) of these $q=2$ Jacobs-like solutions are positive ( 3 and 1), and hence these equilibrium points are local sources.

\section{The case of negligible tilt}

Therefore, neglecting the tilt, and assuming the forms of the potential discussed earlier, we obtain the following system of equations, governing the phenomenological Einstein-aether cosmological models under consideration:

$$
\begin{gathered}
\theta^{2}=3 c^{2} \sigma^{2}+3 \rho_{\phi}-\frac{3}{2} P \\
\dot{\theta}=-\frac{1}{3} \theta^{2}-2 c^{2} \sigma^{2}-\frac{1}{2}\left(\rho_{\phi}+3 p_{\phi}\right)
\end{gathered}
$$




$$
\begin{gathered}
c^{2} \dot{\sigma}=-c^{2} \sigma \theta+\frac{M}{3 \sqrt{3}}\left(\theta^{2}-3 c^{2} \sigma^{2}-3 \rho_{\phi}\right), \\
\ddot{\phi}+\theta \dot{\phi}+V_{\phi}=0 .
\end{gathered}
$$

\section{The scalar field}

Let us now consider the forms of $\rho_{\phi}$ and $p_{\phi}$ in the specific class of geometries under consideration here. The Einstein-aether action, ${ }^{1}$ which is a generalization of the Einstein-Hilbert action, also depends on the aether field (the timelike vector field $u_{a}$ ). In particular, the matter fields can depend on the aether field. The Lagrangian for the matter (scalar) field is given by

$$
L_{\phi}=\frac{1}{2} \nabla_{a} \phi \nabla^{a} \phi-V,
$$

where the self-interaction potential $V$ now can depend on scalar quantities constructed from (the covariant derivative of) $u_{a}$. Taking variations of $V=V(\phi, \theta, \sigma)$ with respect to the metric and the aether field $u_{a}$ (see Eq. (13) in Ref. 1) we obtain (for the models under consideration) the effective density $\rho_{\phi}$ and pressure $p_{\phi}\left(T_{a b}^{\phi} \sim \rho_{\phi} u_{a} u_{b}+p_{\phi} h_{a b}\right)$ of the form (see Appendix B),

$$
\begin{aligned}
\rho_{\phi}= & \frac{1}{2} \dot{\phi}^{2}+V-V_{\theta}(\theta+\sqrt{6} \sigma)-V_{\sigma}\left(\sigma-\frac{1}{\sqrt{6}} \theta\right), \\
p_{\phi}= & \frac{1}{2} \dot{\phi}^{2}-V+V_{\theta}\left(\theta+\sqrt{6} \sigma+\sqrt{6} \frac{\dot{\sigma}}{\theta}\right)+V_{\sigma}\left(\sigma-\frac{1}{\sqrt{6}} \theta-\frac{1}{\sqrt{6}} \frac{\dot{\theta}}{\theta}\right) \\
& +\dot{V}_{\theta}\left(1+\sqrt{6} \frac{\sigma}{\theta}\right)+\dot{V}_{\sigma}\left(\frac{\sigma}{\theta}-\frac{1}{\sqrt{6}}\right) .
\end{aligned}
$$

Note that in the Bianchi I case with $V_{\sigma}=0$, we have that

$$
\begin{aligned}
& \rho_{\phi}=\frac{1}{2} \dot{\phi}^{2}+V-V_{\theta}(\theta+\sqrt{6} \sigma), \\
& p_{\phi}=\frac{1}{2} \dot{\phi}^{2}-V+\theta V_{\theta}+\dot{V}_{\theta}\left(1+\sqrt{6} \frac{\sigma}{\theta}\right) .
\end{aligned}
$$

(In the absence of shear $(\sigma=0)$ we obtain Eq. (14) in Ref. 1).

\section{Special cases}

There are a number of special cases that might be of interest.

- $V=V(\phi, \theta)$, which has non-trivial corrections when the shear is not zero.

- The Bianchi type I subcase with $K=0$.

- The case $c^{2}=1$

Regarding this last subcase, there are 4 dimensionless free parameters (constants) that define the Einstein aether theory $\left(c_{i} ; i=1 \ldots 4\right)$. Since we have normalized $G\left(c_{1}+3 c_{2}+c_{3}=0\right)$, and since there is an invariance in the action in the cosmological application, there are effectively only 2 independent parameters, ${ }^{1,23}$ denoted here by $c^{2}$ and $d$. The arbitrary parameter $d \sim c_{1}-c_{4}$ does not occur in the tilt-free equations above. The final parameter, $c^{2}-1 \sim\left(c_{1}+c_{3}\right)$ satisfies $0 \leq c^{2} \leq 1$, where $c^{2}=1$ is the corresponding GR value. In Ref. 23 it was shown that $\left(c_{1}+c_{3}\right)$ is positive and bounded above; indeed, it is expected that $\left(c_{1}+c_{3}\right)$ is very small (i.e., $\left.\sim 10^{-3}\right)$. Therefore, $c^{2} \sim 1$ is consistent with Refs. 1 and 23. In addition, since it is not expected that there would be any significant different qualitative behaviour in the late time dynamics, we could assume self-consistently here that $c^{2} \sim 1$ to simplify the analysis. 


\section{E. Dimensionless variables}

We now introduce new expansion-normalized variables and a new time variable as follows:

$$
\begin{array}{rlrl}
\beta & =\sqrt{6} \frac{\sigma}{\theta}, & & \frac{d t}{d \tau}=\frac{3}{\theta}, \\
\Psi=\frac{\sqrt{3}}{\sqrt{2}} \frac{\dot{\phi}}{\theta}, & \Phi & =\sqrt{3 V_{0}} \frac{\mathrm{e}^{-\lambda \phi / 2}}{\theta} .
\end{array}
$$

For a given potential $V(\theta, \phi, \sigma)$, we can find $3 \rho_{\phi} / \theta^{2}$ and $3 p_{\phi} / \theta^{2}$, in terms of $\beta, \Psi$, and $\Phi$, and determine the system of differential (evolution) equations in terms of the expansion-normalized variables (see below). We define $K \equiv-\frac{3 P}{2 \theta^{2}}$, and since $K$ is always positive the generalized Friedmann equation usually determines a compact region of interest (i.e., $\beta, \Psi$, and $\Phi$ are bounded). The deceleration parameter, $q$, is defined by the decoupled evolution equation for the expansion

$$
\frac{d \theta}{d \tau}=-\theta(1+q)
$$

and inflation is defined by $q<0$.

\section{A CLASS OF ANISOTROPIC MODELS}

We are looking for general scale invariant solutions in which

$$
V(\theta, \phi, \sigma)=V_{0} e^{-\sqrt{6} \bar{\lambda} \phi}+\frac{a}{\sqrt{3}} \sqrt{V_{0}} \theta e^{-\frac{\sqrt{6} \bar{\lambda} \phi}{2}}+\sqrt{2} b \sqrt{V_{0}} \sigma e^{-\frac{\sqrt{6}}{2} \bar{\lambda} \phi},
$$

where we have defined $\bar{\lambda}=\lambda / \sqrt{6}$, and we have normalized the constants $a$ and $b$ appropriately.

Let us now consider the class of anisotropic models under consideration (with with $B_{1}=1$ and $B_{2}=1$ in Appendix B). The evolution equations are

$$
\begin{aligned}
& \beta^{\prime}=(q-2) \beta-\frac{\sqrt{2} M}{c^{2}} K, \\
& \Psi^{\prime}=(q-2) \Psi+\frac{3 \bar{\lambda}}{2}\left(2 \Phi^{2}+a \Phi+b \beta \Phi\right), \\
& \Phi^{\prime}=(q+1) \Phi-3 \bar{\lambda} \Psi \Phi,
\end{aligned}
$$

where

$$
\begin{aligned}
K & =\frac{c^{2}}{2} \beta^{2}+\Omega-1, \\
\Omega & =\Psi^{2}+\Phi^{2}-a \Phi \beta+b \Phi \\
q-2 & =\frac{\left(\frac{a \sqrt{2} M}{c^{2}} \Phi-4\right) K+3(a+b) \lambda \Psi \Phi \beta+3(a-b) \lambda \Psi \Phi+6 \Phi^{2}+3(b-a \beta) \Phi}{b \Phi-2} .
\end{aligned}
$$

Note that $0 \leq \Omega \leq 1$ and $\frac{c^{2}}{2} \beta^{2} \leq 1$. First we shall make some general comments about the full 3D system. In Sec. IV, we shall comprehensively study the 2D zero curvature case.

\section{A. Equilibrium points with zero curvature $(K=0)$}

\section{One parameter family, $P_{j}$ :}

$$
\Phi=0, \quad \Psi^{2}+\frac{c^{2}}{2} \beta^{2}=1, \quad q_{P_{j}}=2 .
$$


Eigenvalues:

$$
\lambda_{1}=4-\sqrt{2} M \beta, \quad \lambda_{2}=3(1-\bar{\lambda} \Psi), \quad \lambda_{3}=0 .
$$

Since $-1<M<1$, we have that the smallest possible value for $\lambda_{1}$ occurs for $M \rightarrow \pm 1$ when $\Psi$ $=0$ and $\beta= \pm \frac{\sqrt{2}}{|c|}$. On the other hand, the smallest possible value for $\lambda_{2}$ is obtained for the largest possible value of $\Psi$. We have that if $|c|>\frac{1}{2}$ and $\bar{\lambda}<1$, then all of the circle of equilibrium points defined above consists of sources for all allowed values of $M$. If $\bar{\lambda}>1$ or $|c|<\frac{M}{2}$, a part of the circle consists of saddles (or even sinks if $\bar{\lambda}$ is large enough, $M$ is non-zero and $c$ is small enough). The vacuum solution $\Psi=0, M=0, \beta= \pm \frac{\sqrt{2}}{|c|}$ is always a source. These sources correspond to analogues of the (non-oscillatory) Jacobs anisotropic Bianchi I non-vacuum solutions. ${ }^{13}$

\section{Isotropic equilibrium points $p_{2}, p_{3}$ :}

$$
\Phi=\frac{2\left(2 \bar{\lambda}^{2} b-b-a \bar{\lambda}^{2} \pm \sqrt{\left.b^{2}-2 a \bar{\lambda}^{2} b+a^{2} \bar{\lambda}^{2}+4-4 \bar{\lambda}^{2}\right)}\right.}{4 \bar{\lambda}^{2} b^{2}-4 a \bar{\lambda}^{2} b+a^{2} \bar{\lambda}^{2}+4}, \quad \beta=0 .
$$

For physical solutions we require $\Phi$ to be real and positive. To make the linearization (for small values of the parameters $a$ and $b$ ) of the two isotropic equilibrium points $p_{2}, p_{3}$ easier we introduce new parameters $(A, B)$ specifically adapted to the points $p_{2}$ and $p_{3}$ through the relations:

$$
\begin{gathered}
a=\frac{1-\lambda^{2}-A^{2}\left(1+B^{2}\right)}{A}+B\left(\lambda^{-1}-2 \lambda\right), \quad b=\frac{1-\lambda^{2}-A^{2}\left(1+B^{2}\right)-2 A B \lambda}{A}, \\
A=\frac{2\left(2 \bar{\lambda}^{2} b-b-a \bar{\lambda}^{2} \pm \sqrt{\left.\left(b-a \bar{\lambda}^{2}\right)^{2}+\left(1-\bar{\lambda}^{2}\right)\left(a^{2} \bar{\lambda}^{2}+4\right)\right)}\right.}{\bar{\lambda}^{2}(2 b-a)^{2}+4}, \quad B=\left(\frac{a}{2}-b\right) \bar{\lambda} .
\end{gathered}
$$

The two signs are necessary to cover the whole range of parameter values. We then obtain a new parameterization of the two points, $\left(p_{1}, p_{2}\right) \rightarrow\left(\bar{p}_{1}, \bar{p}_{2}\right)$,

$$
\begin{gathered}
\bar{p}_{2}: \Phi=A, \quad \Psi=A B+\bar{\lambda}, \quad \beta=0, \quad q_{\bar{p}_{2}}=-\left(1-3 A B \bar{\lambda}-3 \bar{\lambda}^{2}\right), \quad(A>0), \\
\bar{p}_{3}: \Phi=\frac{\bar{\lambda}^{2}-1}{A\left(B^{2}+1\right)}, \quad \Psi=B \frac{\bar{\lambda}^{2}-1}{A\left(B^{2}+1\right)}+\bar{\lambda}, \quad \beta=0, \\
q_{\bar{p}_{3}}=-\frac{A\left(1+B^{2}\right)\left(1-3 \bar{\lambda}^{2}\right)+3 B \bar{\lambda}\left(1-\bar{\lambda}^{2}\right)}{A\left(1+B^{2}\right)}, \quad\left(A>0, \bar{\lambda}^{2}>1\right), \quad\left(A<0, \bar{\lambda}^{2}<1\right) .
\end{gathered}
$$

Eigenvalues:

$$
\begin{gathered}
\bar{p}_{2}: \quad \lambda_{1}=-3\left(1-A B \bar{\lambda}-\bar{\lambda}^{2}\right), \quad \lambda_{2}=-2\left(1-3 A B \bar{\lambda}-3 \bar{\lambda}^{2}\right), \\
\lambda_{3}=\frac{-3\left(1+A^{2}+A^{2} B^{2}-\bar{\lambda}^{2}\right)}{1+A^{2}+(A B+\bar{\lambda})^{2}}, \\
\bar{p}_{3}: \quad \lambda_{1}=-2 \frac{A\left(1+B^{2}\right)\left(1-3 \bar{\lambda}^{2}\right)+3 B \bar{\lambda}\left(1-\bar{\lambda}^{2}\right)}{A\left(1+B^{2}\right)}, \quad \lambda_{2}=-\frac{3\left(A+A B^{2}+B \bar{\lambda}\right)\left(1-\bar{\lambda}^{2}\right)}{A\left(1+B^{2}\right)}, \\
\lambda_{3}=\frac{-3\left(1-\bar{\lambda}^{2}\right)\left(1-\bar{\lambda}^{2}+A^{2}+A^{2} B^{2}\right)}{\left(1-A B \bar{\lambda}-\bar{\lambda}^{2}\right)^{2}+A^{2}\left(1+B^{2}+\bar{\lambda}^{2}\right)} .
\end{gathered}
$$

The equilibrium point $\bar{p}_{2}$ is an inflationary sink for small $\bar{\lambda}$ and positive $\mathrm{A}$, while $\bar{p}_{3}$ is an inflationary sink for small $\bar{\lambda}$ and negative A.

\section{Anisotropic points, $p_{4}, p_{5}$ :}

$$
\begin{gathered}
\Psi=\frac{1}{\bar{\lambda}}, \quad \Phi=-\frac{1}{2}(b \beta+a), \quad \beta=\frac{b^{2}-a^{2}-a b}{2 b a+2 c^{2}+b^{2}} \pm \\
\frac{\sqrt{\left(\left(a^{2}+b^{2}\right)^{2}+2(2 b-a) a c^{2}\right) \bar{\lambda}^{2}+4\left(2 a b+b^{2}+2 c^{2}\right)\left(\bar{\lambda}^{2}-1\right)}}{\bar{\lambda}\left(2 b a+2 c^{2}+b^{2}\right)} \\
q_{p_{4}, p_{5}}=2 .
\end{gathered}
$$


The non-vacuum solutions corresponding to these equilibrium points also are spatially flat anisotropic solutions with a deceleration parameter equal to 2 (and hence are never inflationary), but with a fraction of the energy density contained in the potential energy of the scalar field.

Eigenvalues:

$$
\lambda_{1}=4-\sqrt{2} M \beta_{p_{4}}, \quad \lambda_{2,3}=-\frac{3 \Phi_{p_{4}, p_{5}}\left(b \beta_{p_{4}, p_{5}}^{2}-b \beta_{p_{4}, p_{5}}+2 \beta_{p_{4}, p_{5}} \Phi_{p_{4}, p_{5}}+b \pm D\right)}{2\left(b \Phi_{p_{4}, p_{5}}-2\right)},
$$

where

$$
\begin{aligned}
D & =\left[\left(-\beta_{p_{4}, p_{5}}\left(b-2 \Phi_{p_{4}, p_{5}}\right)+b \beta_{p_{4}, p_{5}}^{2}+b\right)^{2}-2\left(b \Phi_{p_{4}, p_{5}}-2\right)\left(\beta _ { p _ { 4 } , p _ { 5 } } \overline { \lambda } ^ { 2 } \left(b^{2}+2 b \Phi_{p_{4}, p_{5}}\right.\right.\right. \\
& \left.\left.\left.+4 \Phi_{p_{4}, p_{5}}^{2}\right)+b^{2} \beta_{p_{4}, p_{5}}^{3} \bar{\lambda}^{2}+4 b \beta_{p_{4}, p_{5}}^{2} \bar{\lambda}^{2} \Phi_{p_{4}, p_{5}}+4\left(b \bar{\lambda}^{2} \Phi_{p_{4}, p_{5}}+\bar{\lambda}^{2} \Phi_{p_{4}, p_{5}}^{2}-\bar{\lambda}^{2}+1\right)\right)\right]^{1 / 2} .
\end{aligned}
$$

The signs of the eigenvalues can be studied. In Sec. IV, we will analyse the equilibrium points in the zero curvature case in detail. There are some sources, which are analogues of the Jacobs solutions studied above.

\section{B. Equilibrium points with curvature}

$$
\begin{gathered}
p_{6}: \quad \Phi=0, \quad \Psi=0, \quad \beta=\frac{M}{\sqrt{2} c^{2}}, \\
K=\frac{M^{2}}{4 c^{2}}-1, \quad q=\frac{M^{2}}{2 c^{2}} .
\end{gathered}
$$

Eigenvalues:

$$
\lambda_{1}=\frac{M^{2}}{2 c^{2}}+1, \quad \lambda_{2}=\frac{M^{2}}{2 c^{2}}-2, \quad \lambda_{3}=\frac{M^{2}}{2 c^{2}}-2 .
$$

This equilibrium point is always a saddle when the curvature $K$ is negative.

\section{Discussion}

There are a number of important invariant sets. From Eqs. (B3)-(B5), we can compute the evolution equations for $K$. By setting $K=0$ in this expression we obtain $K^{\prime}=0$, so that $K=0$ is an invariant set of the system. We recall that we can obtain the Bianchi I models (with $K=0$ ) when $M$ $=N=0$. We shall study this $2 \mathrm{D}$ invariant set (not necessarily for small values of the parameters $a$ and $b$ ) in Sec. IV (where we will use the same notation for the 2D equilibrium points as the 3D zero curvature equilibrium points in this section).

In the invariant set $\Phi=0$, the scalar potential is dynamically negligible. The isotropic models with $\beta=0$ (i.e., no shear anisotropy), were studied in Ref. 20.

\section{ANALYSIS OF THE SYSTEM WITH ZERO CURVATURE}

When $K=0$ we obtain the 2D system (we also set $c^{2}=1$ ),

$$
\begin{aligned}
& \beta^{\prime}=(q-2) \beta, \\
& \Phi^{\prime}=(q+1) \Phi-3 \bar{\lambda} \Psi \Phi,
\end{aligned}
$$

where

$$
\begin{gathered}
\Psi^{2}=1-\frac{1}{2} \beta^{2}-\Phi^{2}+a \beta \Phi-b \Phi \\
q-2=\frac{3 \Phi}{b \Phi-2}[(\bar{\lambda} \Psi-1)(a+b) \beta+(a-b) \bar{\lambda} \Psi+b(\beta+1)+2 \Phi] .
\end{gathered}
$$


TABLE I. Equilibrium points of the anisotropic model.

\begin{tabular}{lcc}
\hline \hline Eq pts & $\Phi$ & $\beta$ \\
\hline$p_{2,3}$ & $\frac{2\left(2 \bar{\lambda}^{2} b-b-a \bar{\lambda}^{2} \pm Z\right)}{4 \bar{\lambda}^{2} b^{2}-4 a \bar{\lambda}^{2} b+a^{2} \bar{\lambda}^{2}+4}$ & 0 \\
$p_{4,5}$ & $-\frac{-\bar{\lambda} b^{3}-b a^{2} \bar{\lambda}-2 a \bar{\lambda} \pm b N}{2 \bar{\lambda}\left(2+2 a b+b^{2}\right)}$ & $\frac{\bar{\lambda} b^{2}-\bar{\lambda} a b-\bar{\lambda} a^{2} \mp N}{\bar{\lambda}\left(2+2 a b+b^{2}\right)}$ \\
\hline \hline
\end{tabular}

\section{A. Equilibrium points}

We define

$$
Z=\sqrt{b^{2}-2 a b \bar{\lambda}^{2}+a^{2} \bar{\lambda}^{2}+4-4 \bar{\lambda}^{2}}
$$

and

$$
N^{2}=b^{4} \bar{\lambda}^{2}+2 \bar{\lambda}^{2} a^{2} b^{2}+\bar{\lambda}^{2} a^{4}+8 \bar{\lambda}^{2}-8+12 a b \bar{\lambda}^{2}-2 a^{2} \bar{\lambda}^{2}-8 a b+4 b^{2} \bar{\lambda}^{2}-4 b^{2} .
$$

The equilibrium points that are potentially of interest (i.e., possible future attractors) in the $2 \mathrm{D}$ system with zero curvature are given in Table I.

For physical solutions all of $\Phi, \beta$, and $\Psi$ must be real, and $\Phi \geq 0$. Since $K=0$, if $\Omega \geq 0$, then $\Omega$ and $\beta^{2}$ are bounded. For sufficiently small $\bar{\lambda}, Z$ is real. We cannot hope to study the stability of these equilibrium points in all generality. However, we consider some appropriate values of $\bar{\lambda}$ (e.g., for small $\bar{\lambda}$ such as $\bar{\lambda}=\frac{1}{2}$ ). First, if $a=b=0$ we will have the same equilibrium points $p_{i}$ as in the single scalar field model with matter. We will study the stability of the $p_{i}$ in the two cases $a=0$ and $b=0$, separately.

For the point $p_{1}$, where $\Phi=0$ and $\beta= \pm \frac{\sqrt{2 \bar{\lambda}^{2}-2}}{\bar{\lambda}}$, which is only valid for $\bar{\lambda}>1$, we have that $q=2$, and hence $p_{1}$ is always non-inflationary. $p_{1}$ always has one zero eigenvalue. In general, this point will be a source, and we will not consider it further here. Some of the other equilibria may also turn out to be sources for particular ranges of the parameters. Recall that analogues of the Jacobs anisotropic Bianchi I non-vacuum solutions are sources.

\section{Point $p_{2}$}

$\Phi$ is always positive for sufficiently small $\bar{\lambda}$. The value of $\Psi$ for $p_{2}$ is given by

$$
\Psi=\frac{\bar{\lambda}\left(-a b+2 b^{2}+4+a Z-b Z\right)}{4 \bar{\lambda}^{2} b^{2}-4 a \bar{\lambda}^{2} b+a^{2} \bar{\lambda}^{2}+4} .
$$

We study the stability in two cases for the values of $a, b$ between -3 and 3 .

The first case is when $a=0$. For $a=0, \Phi$ is real and positive for $\bar{\lambda}^{2}<1$, and also for $\bar{\lambda}^{2}>1$ if $b>2 \sqrt{\bar{\lambda}^{2}-1}>0$. The eigenvalues $\mu_{i}$, where $i=1,2$ (corresponding to,+- ), are given by

$$
\mu_{i}=\frac{\left.-3\left(m_{2} m_{1}\left(m_{3}+m_{4}\right)\right) \pm \sqrt{2} m_{1} m_{5}+\sqrt{2} \bar{\lambda}^{3} b^{2} m_{6}\right)}{m_{2}\left(m_{7} \pm m_{1} m_{8}\right)},
$$

where

$$
\begin{gathered}
m_{1}=\sqrt{b^{2}-4 \bar{\lambda}^{2}+4}, \\
m_{2}=\sqrt{\frac{-\bar{\lambda}^{2}\left(-4 b^{2}+2 \bar{\lambda}^{2} b^{2}+\left(b^{3}+2\right) b m_{1}-2-b^{4}\right)}{\left(\bar{\lambda}^{2} b^{2}+1\right)^{2}}}, \\
m_{3}=4 b \bar{\lambda}^{2}+4 b^{3} \bar{\lambda}^{4}+b^{5} \bar{\lambda}^{4}-b,
\end{gathered}
$$




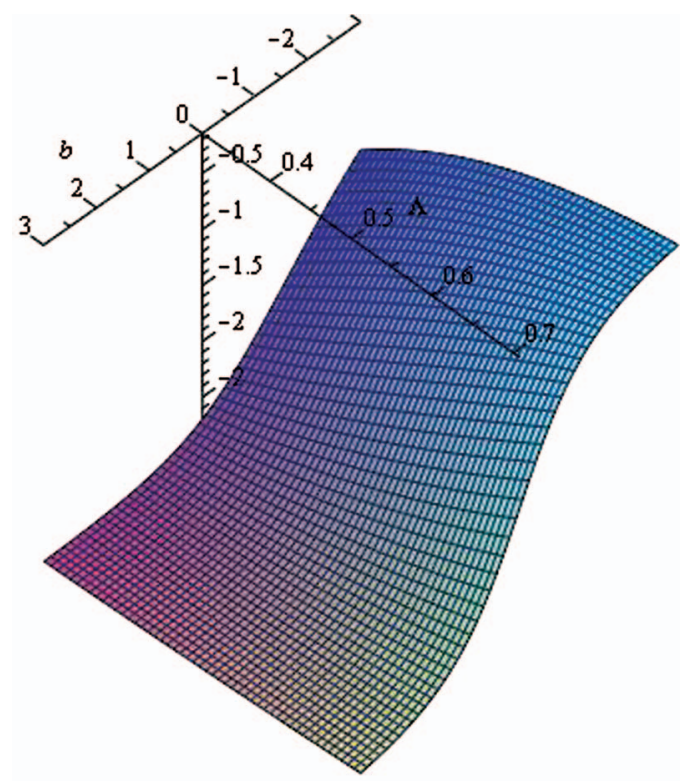

FIG. 1. $a=0 ; \mu_{1}$-eigenvalue of $p_{2}$.

$$
\begin{gathered}
m_{4}=4-b^{6} \bar{\lambda}^{4}+b^{2}-4 \bar{\lambda}^{2}+4 b^{4} \bar{\lambda}^{6} 2 \bar{\lambda}^{2} b^{6}+2 b^{2} \bar{\lambda}^{2} \\
m_{5}=b^{5} \bar{\lambda}^{5}+3 b^{3} \bar{\lambda}^{5}-3 b^{3} \bar{\lambda}^{3}-b \bar{\lambda}^{3}-b^{3} \bar{\lambda}^{5} \\
m_{6}=5+b^{4}-b^{4} \bar{\lambda}^{2}+5 b^{2}+2 b^{2} \bar{\lambda}^{4}-6 \bar{\lambda}^{2}-7 b^{2} \bar{\lambda}^{2} \\
m_{7}=10 b^{2} \bar{\lambda}^{2}+b^{6} \bar{\lambda}^{4}+2 b^{4} \bar{\lambda}^{2}+2 b^{6} \bar{\lambda}^{6}+8 b^{4} \bar{\lambda}^{4}+4+b^{2} \\
m_{8}=b^{5} \bar{\lambda}^{4}+2 b^{3} \bar{\lambda}^{2}+b .
\end{gathered}
$$

We plot the two eigenvalues $\mu_{1}, \mu_{2}$, where the horizontal axes are the values of the parameters $(b, \bar{\lambda})$ (where $\Lambda$ in Figures 1 and 2 is equal to $\bar{\lambda}$ ), and the vertical axes are the values of $\mu_{1}, \mu_{2}$.

For illustration, we can also evaluate the two eigenvalues when $\bar{\lambda}=\frac{1}{2}$. We plot the two eigenvalues $\mu_{1}, \mu_{2}$ when $\bar{\lambda}=\frac{1}{2}$ in Figs. 3 and 4 (where the horizontal axes are the values of $-3 \leq b \leq 3$ and the vertical axes are the value of the eigenvalues $\left.\mu_{1}, \mu_{2}\right)$. As can be seen from the figures, for the parameter $-3 \leq b \leq 3$, we have two negative eigenvalues which implies that $p_{2}$ is a sink.

The second case is when $b=0 . \Phi$ is real and positive if $\bar{\lambda}^{2} \leq 1$ and $a<2$ (including negative values of $a$ ) or $\bar{\lambda}^{2}>1$ and $a>2$. The eigenvalues are given by

$$
\mu_{i}=\frac{3\left(m_{13} m_{15} \pm m_{14} m_{16}+m_{17}\right)}{m_{14}\left(48 a^{2} \bar{\lambda}^{2}+12 a^{4} \bar{\lambda}^{4}+a^{6} \bar{\lambda}^{6}+64\right)},
$$

where

$$
\begin{gathered}
m_{13}=\sqrt{4-4 \bar{\lambda}^{2}+\bar{\lambda}^{2} a^{2}}, \\
m_{14}=\sqrt{\frac{\bar{\lambda}^{2}\left(4 a^{2}+a^{4} \bar{\lambda}^{2}-4 a^{2} \bar{\lambda}^{2}+8 a m_{13}+16\right.}{\left(4+a^{2} \bar{\lambda}^{2}\right)^{2}}} \\
m_{15}=-2 a \bar{\lambda}^{3} m_{13}\left(16+4 a^{2}+a^{2} \bar{\lambda}^{2}-12 \bar{\lambda}^{2}\right),
\end{gathered}
$$




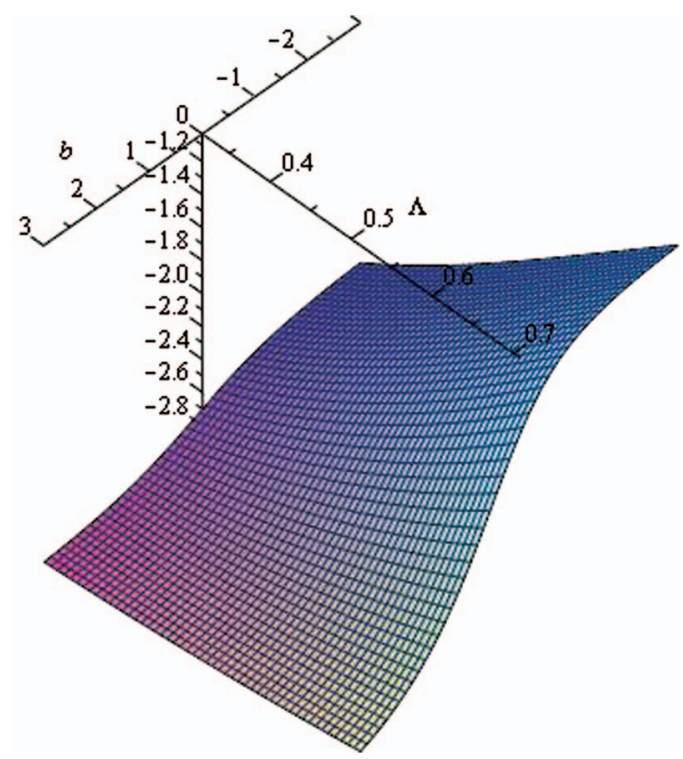

FIG. 2. $a=0 ; \mu_{2}$-eigenvalue of $p_{2}$.

$$
\begin{gathered}
m_{16}=-64-32 a^{2} \bar{\lambda}^{2}-4 a^{4} \bar{\lambda}^{4}+64 \bar{\lambda}^{2}+8 a \bar{\lambda}^{2} m_{13}\left(a^{2} \bar{\lambda}^{2}+4\right)-4 a^{4} \bar{\lambda}^{6}, \\
m_{17}=-32 a^{2} \bar{\lambda}^{3}-4 a^{4} \bar{\lambda}^{5} a^{6} \bar{\lambda}^{7}+48 a^{2} \bar{\lambda}^{5}-4 a^{4} \bar{\lambda}^{7} .
\end{gathered}
$$

We plot the two eigenvalues $\mu_{1}, \mu_{2}$ in Figs. 5 and 6, where the horizontal axes are the values of the parameters $(a, \bar{\lambda})$ (where $\Lambda$ in the figures below is equal to $\bar{\lambda}$ ), and the vertical axes are the values of $\mu_{1}, \mu_{2}$.

For illustration, we can also evaluate the two eigenvalues when $\bar{\lambda}=\frac{1}{2}$. We plot the two eigenvalues $\mu_{1}, \mu_{2}$ when $\bar{\lambda}=\frac{1}{2}$ in Figs. 7 and 8 (where the horizontal axes are the values of $-3 \leq a$

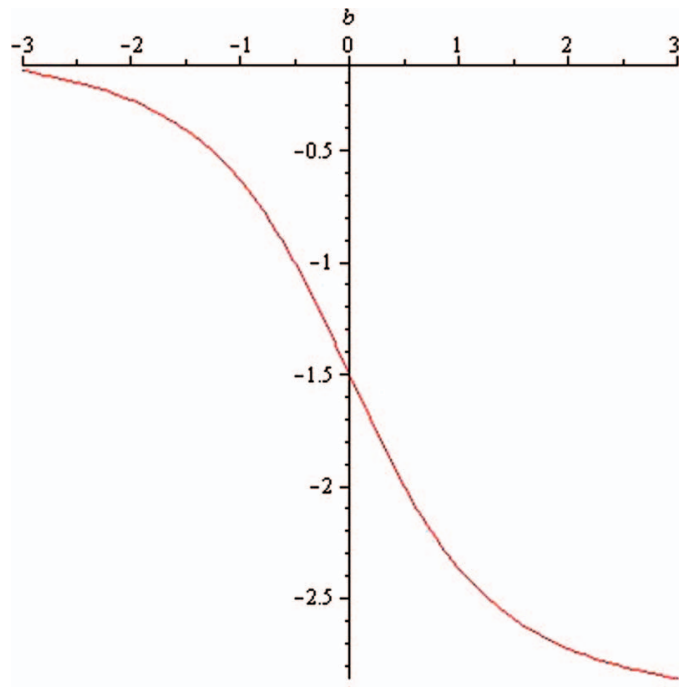

FIG. 3. $\bar{\lambda}=\frac{1}{2}, a=0 ; \mu_{1}$ - plot for $p_{2}$. 


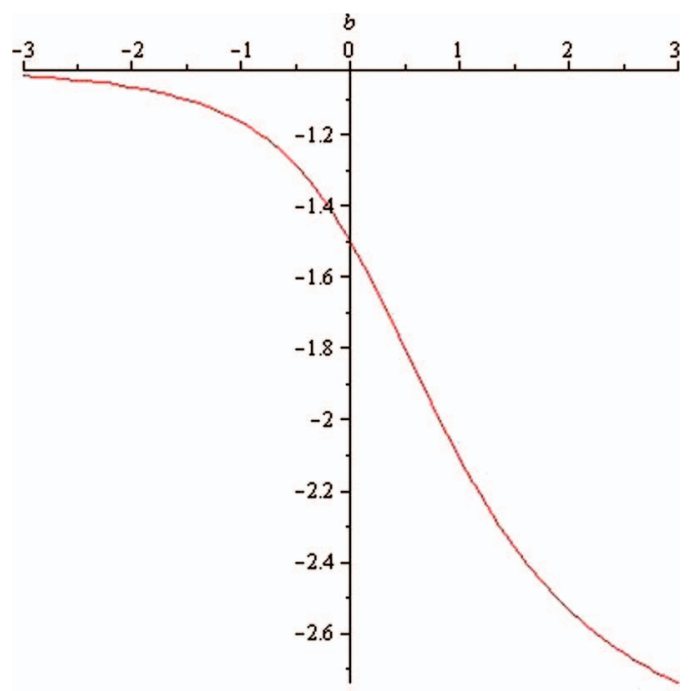

FIG. 4. $\bar{\lambda}=\frac{1}{2}, a=0 ; \mu_{2}$-plot for $p_{2}$.

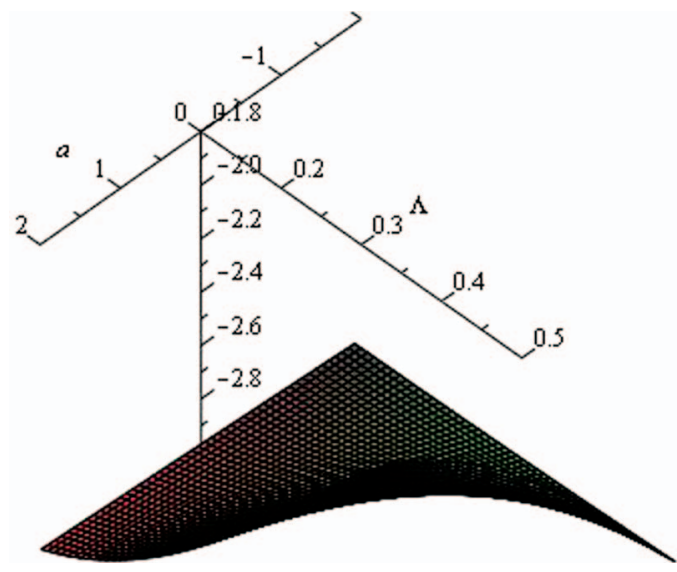

FIG. 5. $b=0 ; \mu_{1}$-eigenvalues of $p_{2}$.

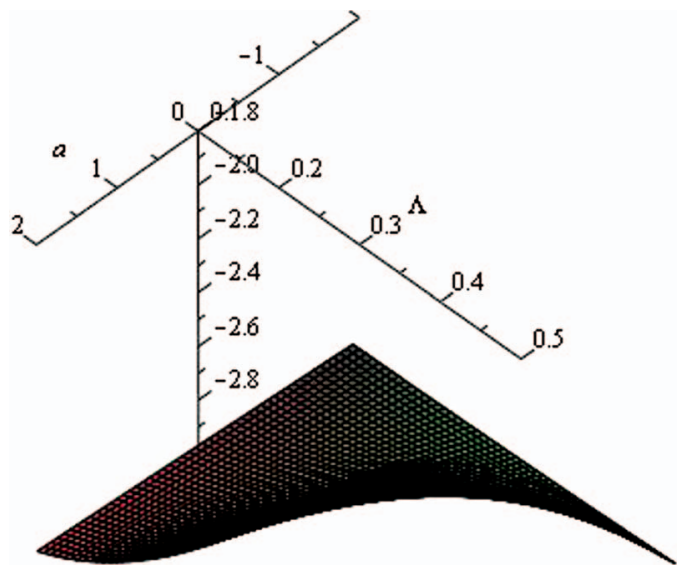

FIG. 6. $b=0 ; \mu_{2}$-eigenvalues of $p_{2}$. 


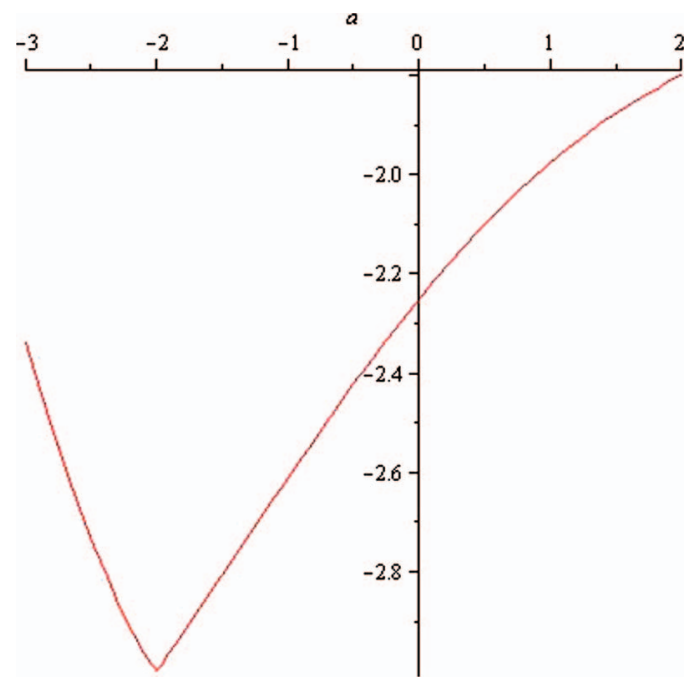

FIG. 7. $\bar{\lambda}=\frac{1}{2}, b=0 ; \mu_{1}$ - plot of $p_{2}$.

$\leq 2$ and the vertical axes are the values of the eigenvalues $\mu_{1}, \mu_{2}$ ). As you can seen from the Figs. 7 and 8 , we always have a sink for the values $-3 \leq a \leq 2$.

The deceleration parameter for $p_{2}$ :

In this case, the deceleration parameter can be written in the form

$$
q=\frac{-4\left(4+b^{2}\right)+\bar{\lambda}^{2}(G+Z C)+Z b}{(X)(S-b Z)}
$$

where

$$
\begin{gathered}
G=-20 a b+12 a^{2} \bar{\lambda}^{2}+36 b^{2}-a^{2} b^{2}-2 a b^{3}-16 a b^{3} \bar{\lambda}^{2}+9 \bar{\lambda}^{2} a^{2} b^{2} \\
+\bar{\lambda}^{2} a^{3} b-8 a^{2}+8 b^{4}+48+4 \bar{\lambda}^{2} b^{4}-\bar{\lambda}^{2} a^{4}
\end{gathered}
$$

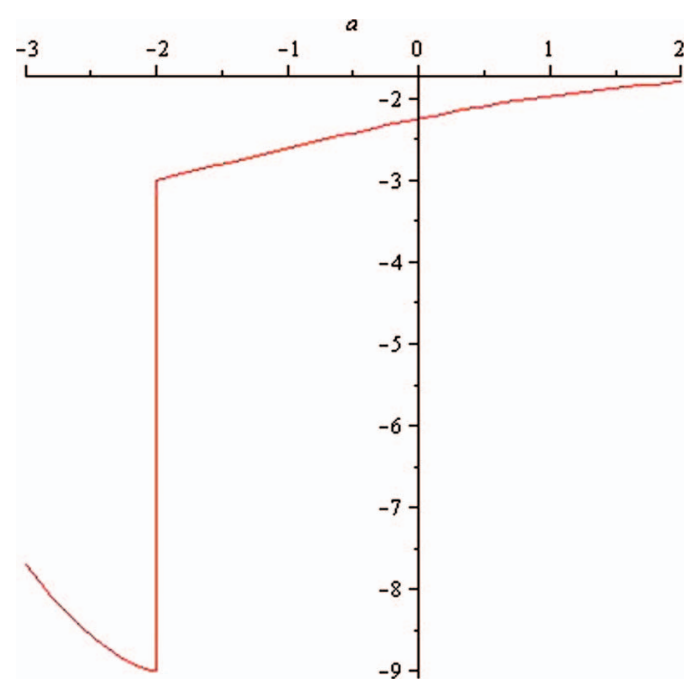

FIG. $8 . \bar{\lambda}=\frac{1}{2}, b=0 ; \mu_{2}$-plot of $p_{2}$. 


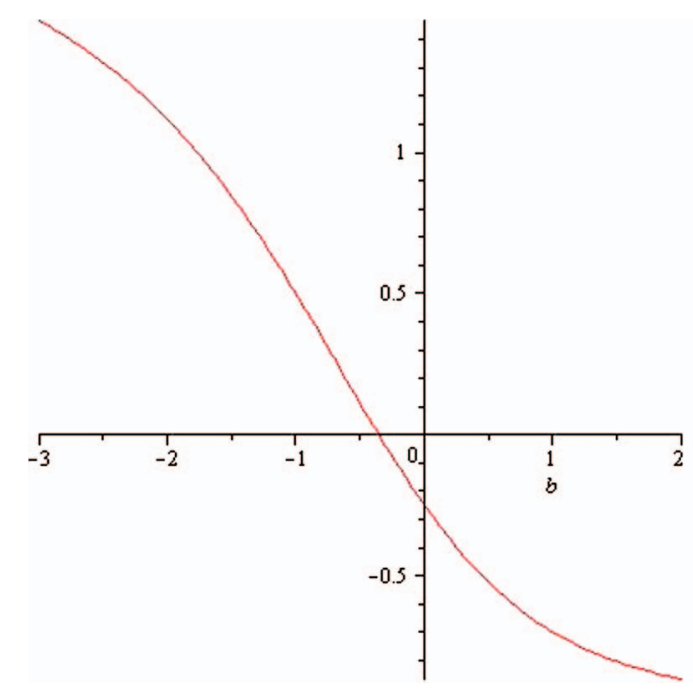

FIG. $9 . \bar{\lambda}=\frac{1}{2}, a=0 ; q$ for $p_{2}$.

$$
\begin{gathered}
C=-8 b^{3}-12 b^{3} \bar{\lambda}^{2}+3 \bar{\lambda}^{2} a^{3}-36 b+12 a+b a 62+2 a b^{2}-15 b a^{2} \bar{\lambda}^{2}-24 \bar{\lambda}^{2} a b^{2}, \\
S=b^{2}+2 b^{2} \bar{\lambda}^{2}-3 a b \bar{\lambda}^{2}+4+a^{2} \bar{\lambda}^{2},
\end{gathered}
$$

and

$$
X=4 \bar{\lambda}^{2} b^{2}-4 a \bar{\lambda}^{2} b+a^{2} \bar{\lambda}^{2}+4
$$

and we note that $Z$ is defined earlier. We consider the inflationary behaviour in the same two cases as in the stability analysis above; i.e, when $a=0$ and $\bar{\lambda}=\frac{1}{2}$ and when $b=0$ and $\bar{\lambda}=\frac{1}{2}$. We plot the values of $q$, where the horizontal axis is the value of $a$ or $b$ and the vertical axis is the value of $q$, in Figs. 9 and 10. For $\bar{\lambda}=\frac{1}{2}$, and the case where $a=0$, if $b \gtrsim-\frac{1}{2}$, then $q$ is negative; otherwise $q$ is positive. For the second case where $b=0$, if $a \gtrsim 1$, then $q$ is positive; otherwise $q$ is negative. That is, the model is inflationary (in the latter case) when $a \lesssim 1$.

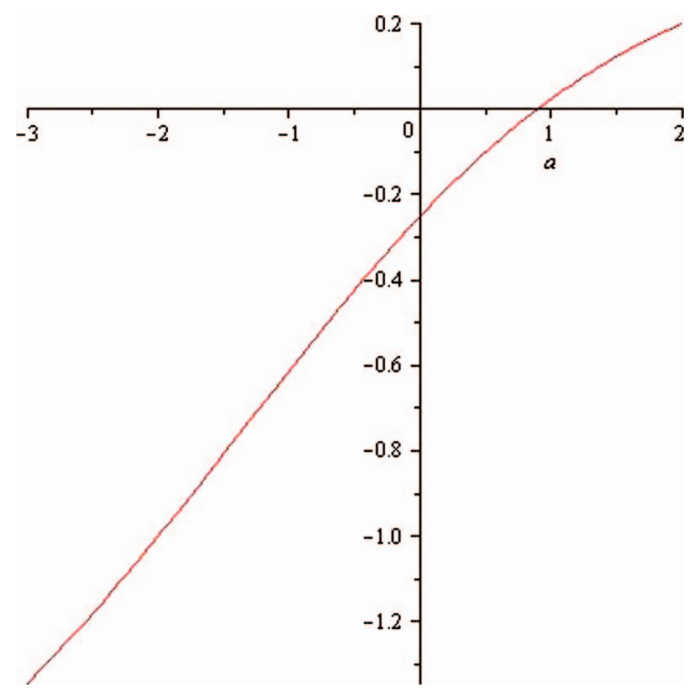

FIG. 10. $\bar{\lambda}=\frac{1}{2}, b=0 ; q$ for $p_{2}$ 


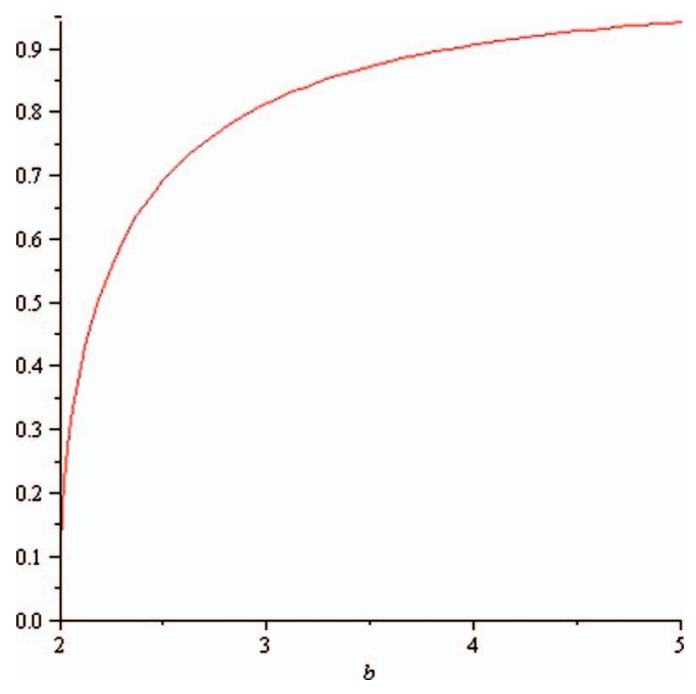

FIG. 11. $\bar{\lambda}=\sqrt{2}, a=0 ; \mu_{1}$-eigenvalues of $p_{3}$.

\section{Point $p_{3}$}

The value of $\Psi$ for $p_{3}$ is given by

$$
\Psi=\frac{\bar{\lambda}\left(-a b+2 b^{2}+4-a Z+b Z\right)}{4 \bar{\lambda}^{2} b^{2}-4 a \bar{\lambda}^{2} b+a^{2} \bar{\lambda}^{2}+4} .
$$

The value of $\Phi$ is given by

$$
\Phi=\frac{2\left(2 \bar{\lambda}^{2} b-b-a \bar{\lambda}^{2}-\sqrt{b^{2}-2 a b \bar{\lambda}^{2}+a^{2} \bar{\lambda}^{2}+4-4 \bar{\lambda}^{2}}\right)}{4 \bar{\lambda}^{2} b^{2}-4 a \bar{\lambda}^{2} b+a^{2} \bar{\lambda}^{2}+4} .
$$

For small $\bar{\lambda}, \Phi$ is negative; e.g., when $a=0$ and $\bar{\lambda}=\frac{1}{2}$,

$$
\Phi=-\frac{b+2 \sqrt{b^{2}+3}}{b^{2}+4},
$$

which is always negative. Indeed, for positive real $\Phi$ we must have that $\bar{\lambda}>1$ and $b>2 \sqrt{\bar{\lambda}^{2}-1}>0$.

The first case is when $a=0$ : the eigenvalues $\mu_{i}$ are given by

$$
\mu_{i}=\frac{-3\left(m_{2}\left(m_{1}\left(m_{3}+m_{4}\right)\right) \mp \sqrt{2} m_{1} m_{5}+\sqrt{2} \bar{\lambda}^{3} b^{2} m_{6}\right)}{m_{2}\left(m_{7} \mp m_{1} m_{8}\right)} .
$$

We plot the two eigenvalues $\mu_{1}, \mu_{2}$ when $\bar{\lambda}^{2}=2$ in Figs. 11 and 12 (where the horizontal axes are the values of $2<b \leq 5$ and the vertical axes are the values of the eigenvalues $\mu_{1}, \mu_{2}$ ). The signs of the two eigenvalues are different, which implies that $p_{3}$ in this case is a saddle.

The second case is when $b=0$; we have

$$
\Phi=\frac{2}{4+a^{2} \bar{\lambda}^{2}}\left(-a \bar{\lambda}^{2}-\sqrt{4+a^{2} \bar{\lambda}^{2}-4 \bar{\lambda}^{2}}\right) .
$$

Thus, $\Phi$ is real and positive when $\bar{\lambda}>1, a<\frac{-2}{\bar{\lambda}} \sqrt{\bar{\lambda}^{2}-1}<0$. The eigenvalues are given by

$$
\mu_{i}=\frac{3\left(m_{13} m_{15} \mp m_{14} m_{16}+m_{17}\right)}{m_{14}\left(48 a^{2} \bar{\lambda}^{2}+12 a^{4} \bar{\lambda}^{4}+a^{6} \bar{\lambda}^{6}+64\right)} .
$$

We plot the two eigenvalues $\mu_{1}, \mu_{2}$ when $\bar{\lambda}^{2}=2$ in Figs. 13 and 14 (where the horizontal axis are the values of $-4 \leq a<-1$ and the vertical axis are the values of the eigenvalues $\mu_{1}, \mu_{2}$ ). We always have positive signs for the both eigenvalues $\mu_{1}$ and $\mu_{2}$ which means that $p_{3}$ is a source in the range of $-4 \leq a<-1$. 


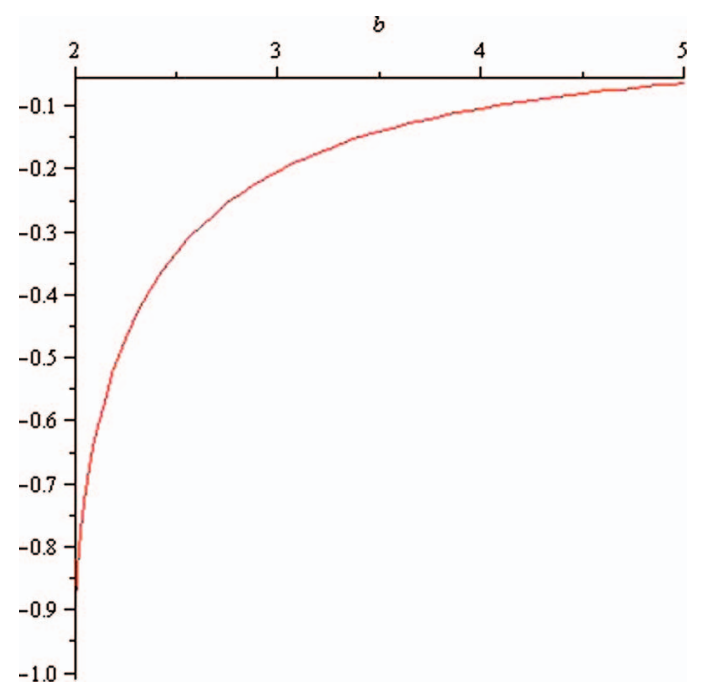

FIG. 12. $\bar{\lambda}=\sqrt{2}, a=0 ; \mu_{2}$-eigenvalues of $p_{3}$.

The deceleration parameter for $p_{3}$ :

In this case, the deceleration parameter can be written in the form

$$
q=\frac{-4\left(4+b^{2}\right)+\bar{\lambda}^{2}(G-Z C)-Z b}{(X)(S+b Z)} .
$$

In the two cases considered above, in which $\bar{\lambda}^{2}=2, p_{3}$ is either a saddle or a source. For illustration, we plot the values of $q$ when $a=0$ and $\bar{\lambda}=\frac{1}{2}$ and when $b=0$ and $\bar{\lambda}=\frac{1}{2}$ in Figs. 15 and 16, where the horizontal axis is the value of $a$ or $b$ and the vertical axis is the value of $q$. When $a=0$, if $b \gtrsim \frac{1}{2}$, then $q$ is positive (otherwise $q$ is negative). When $b=0$, if $a \lesssim-1$, then $q$ is positive (otherwise $q$ is negative).

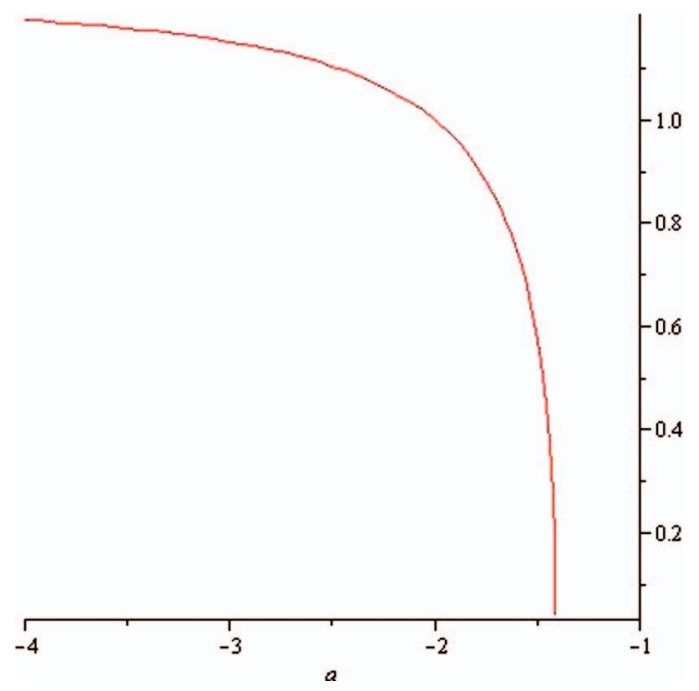

FIG. 13. $\bar{\lambda}=\sqrt{2}, b=0 ; \mu_{1}$-eigenvalues of $p_{3}$. 


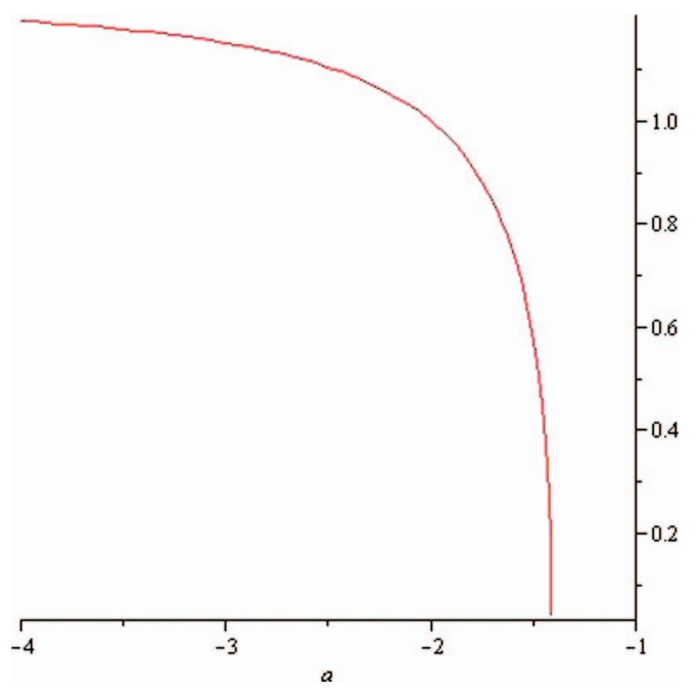

FIG. 14. $\bar{\lambda}=\sqrt{2}, b=0 ; \mu_{2}$-eigenvalues of $p_{3}$.

\section{Point $p_{4}$}

In this case $\Psi=\frac{1}{\bar{\lambda}}$. The value of $\Phi$ is given by

$$
\Phi=\frac{-\bar{\lambda} b^{3}-b a^{2} \bar{\lambda}-2 a \bar{\lambda}-b N}{2 \bar{\lambda}\left(2+2 a b+b^{2}\right)},
$$

where $N$ was defined earlier (before the table for the equilibrium points). $\Phi$ is real if $N>0$ and it is positive if $b<0$. We define

$$
\begin{gathered}
J_{1}=b^{11}-7 b^{9}-32 b^{7}-92 b^{5}-112 b^{3}-32 b, \\
J_{2}=b^{13}-13 b^{11}-20 b^{9}-20 b^{7}-160 b^{5}-416 b^{3}-384, \\
J=-2 b^{20}-4200 b^{14}+15 b^{22}-12576 b^{12}-34704 b^{10}-b^{24}-2 b^{2}\left(1116 b^{16}-189 b^{18}\right),
\end{gathered}
$$

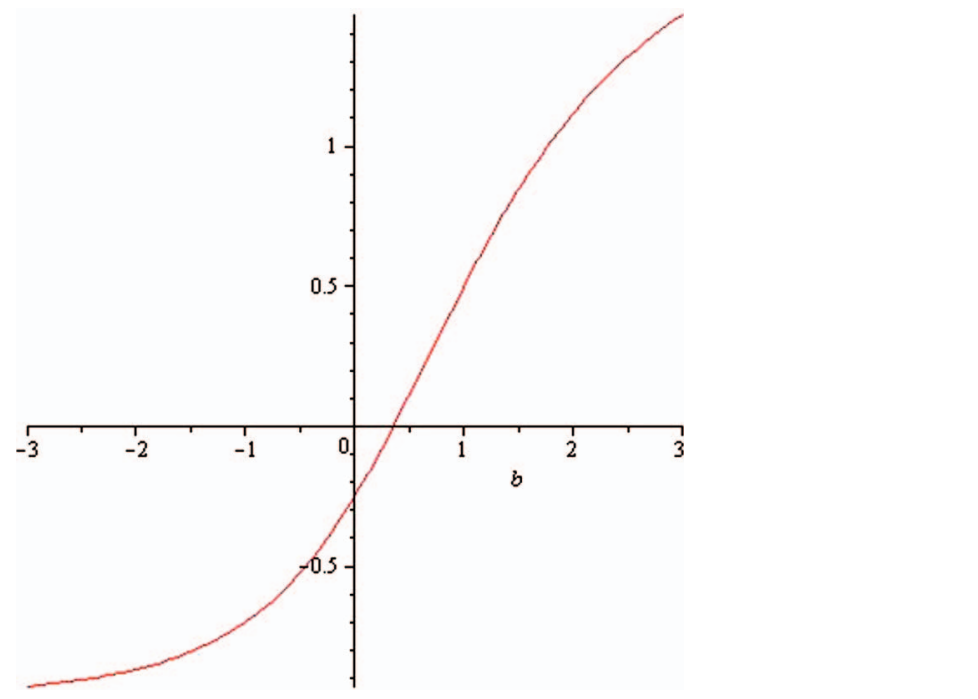

FIG. 15. $\bar{\lambda}=\frac{1}{2}, a=0 ; q$ for $p_{3}$. 


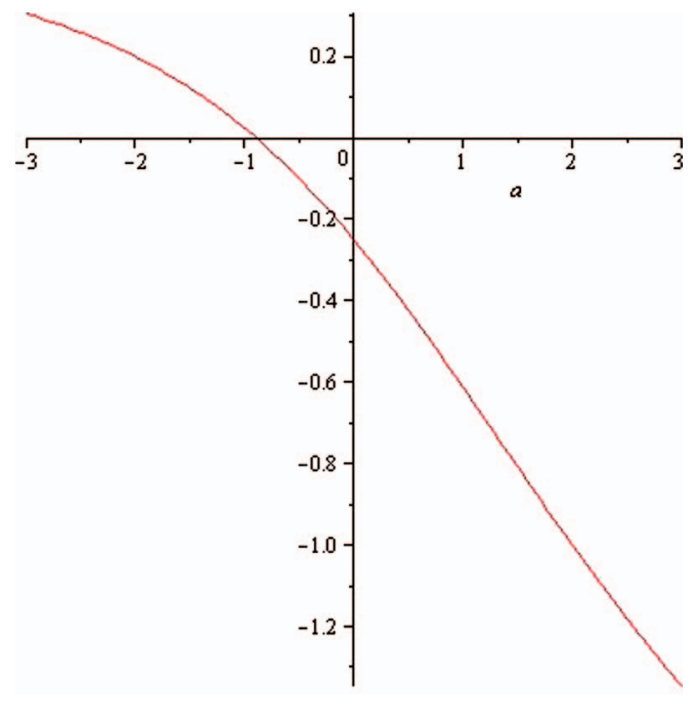

FIG. 16. $\bar{\lambda}=\frac{1}{2}, b=0 ; q$ for $p_{3}$.

$$
\begin{gathered}
\bar{J}=+582784 b^{10}+471552 b^{8}+345984 b^{12}-2 b^{28}+127584 b^{14}, \\
F_{1}=\bar{J}-894 b^{22}-124 b^{24}+42 b^{26}+28128 b^{16}+312 b^{18}, \\
J_{3}=\sqrt{2 b^{2}\left(-164864 b^{2}-205824 b^{4}-61440-82752 b^{8}-156160 b^{6}+J\right)}, \\
J_{4}=\sqrt{-294912-983040 b^{4}-909312 b^{2}-23142 b^{6}+F_{1}}, \\
J_{5}=18 b^{6}+b^{10}+48 b^{4}+48 b^{2}+3 b^{8}, \\
J_{6}=-3 b^{10}-30 b^{8}+b^{12}-80 b^{6}+192 b^{2}+128 .
\end{gathered}
$$

There are also two cases for $p_{4}$. First, when $a=0$ and $\bar{\lambda}=\frac{1}{2}$, the value of $\Phi$ is

$$
\Phi=-\frac{1}{2} b\left(\frac{b^{2}+\sqrt{b^{4}-12 b^{2}-24}}{b^{2}+2}\right),
$$

which is real if $b^{4}-12 b^{2}-24>0$ and $\Phi$ is positive for $b<0$; i.e, we consider $b<-3.75$. The eigenvalues are given by

$$
\mu_{i}=\frac{3 b\left(\sqrt{b^{4}-12 b^{2}-24}\left(J_{1}\right)+J_{2} \pm \sqrt{b^{4}-12 b^{2}-24} J_{3} \pm J_{4}\right)}{2\left(b^{2}+2\right)\left(\sqrt{b^{4}-12 b^{2}-24} J_{5}+J_{6}\right)} .
$$

We note that $\mu_{1}(b)=\mu_{2}(-b)$.

We plot the eigenvalues $\mu_{1}, \mu_{2}$ in Figs. 17 and 18, where the horizontal axis is the real part of the eigenvalues and the vertical axis is the imaginary part of the eigenvalues $\mu_{1}$ and $\mu_{2}$ for the range of the parameter $-7 \leq b \leq-4$. As can be seen from these figures, the real parts of $\mu_{1}$ and $\mu_{2}$ are both positive in the range $-7 \leq b \leq-4$, so that $p_{4}$ is a source.

In the second case, when $b=0$, the value of $\Phi$ is

$$
\Phi=-\frac{a}{2}
$$




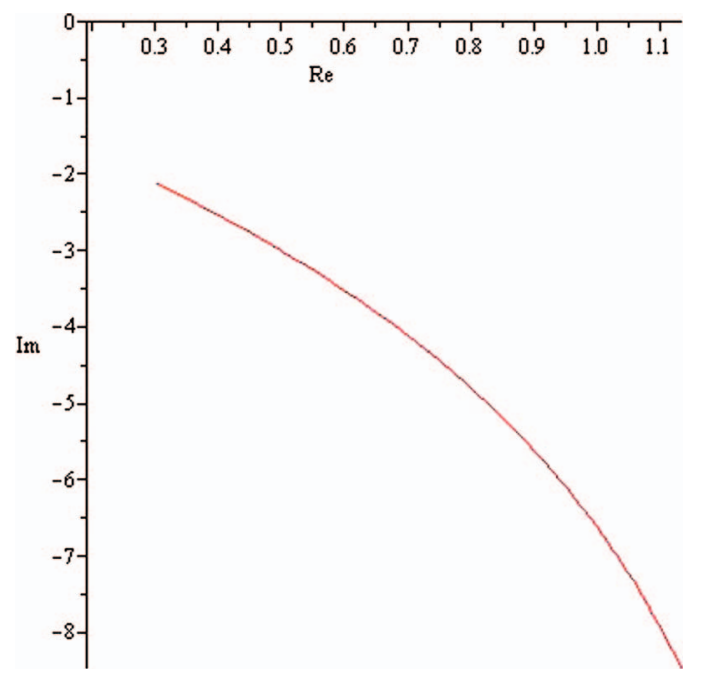

FIG. 17. $\bar{\lambda}=\frac{1}{2}, a=0 ; \mu_{1}$-eigenvalue of $p_{4} ;$ Plot $-7 \leq b \leq-4$.

which is valid if $a<0$. Let now consider the case when $b=0$ and $\bar{\lambda}=\frac{1}{2}$; the eigenvalues are given by

$$
\mu_{i}=\frac{3}{16} a\left(-a^{3}+a n_{2} \pm \overline{n_{2}}\right)
$$

where

$$
\begin{gathered}
n_{2}=\sqrt{\left(a^{2}-6\right)\left(a^{2}+4\right)}, \\
\overline{n_{2}}=\sqrt{2 a^{6}-2 a^{4} n_{2}-20 a^{2}-4 a^{4}+48+2 a^{2} n_{2}} .
\end{gathered}
$$

Again we note that $\mu_{2}(a)=\mu_{1}(-a)$. We plot the eigenvalues above in Figs. 19 and 20 (where the horizontal axis is the real part of the eigenvalues and the vertical axis is the imaginary part of the eigenvalues for the range of parameter $-3 \leq a<0$ ). As can be seen from these figures, the real

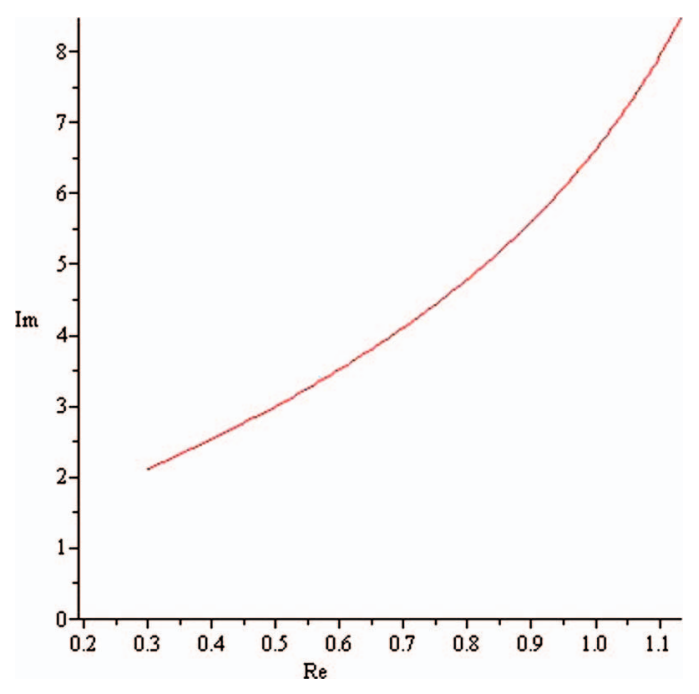

FIG. 18. $\bar{\lambda}=\frac{1}{2}, a=0 ; \mu_{2}$-eigenvalue of $p_{4} ;$ Plot $-7 \leq b \leq-4$. 


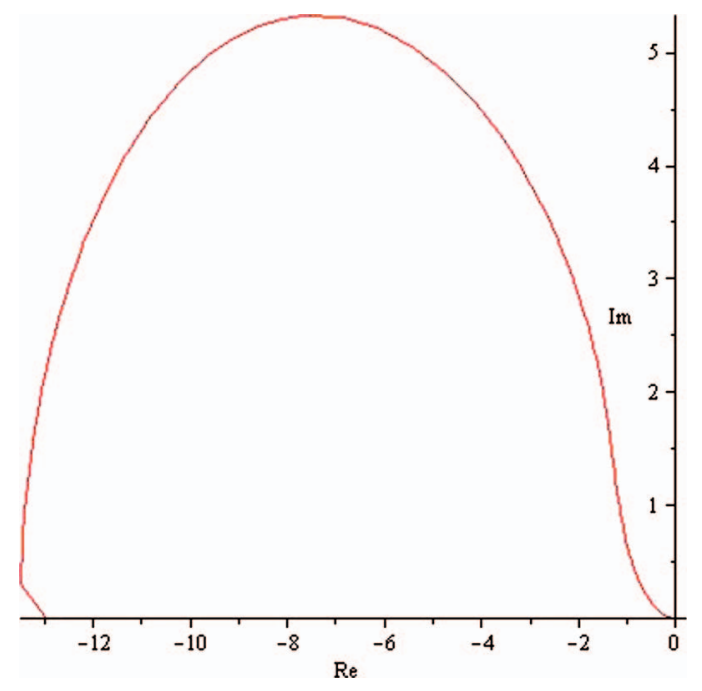

FIG. 19. $\bar{\lambda}=\frac{1}{2}, b=0 ; \mu_{1}$-eigenvalue of $p_{4}$; Plot for $-3 \leq a<0$.

part of $\mu_{1}$ is negative and the real part of $\mu_{2}$ is positive in the range $-3 \leq a<0$, which means that in this case $p_{4}$ is a saddle.

\section{Point $p_{5}$}

The first case for $p_{5}$ is when $a=0$. The value of $\Phi$ is given by

$$
\Phi=\frac{-\bar{\lambda} b^{3}+b \sqrt{D}}{2 \bar{\lambda}\left(2+b^{2}\right)},
$$

where $D \equiv b^{4} \bar{\lambda}^{2}+8 \bar{\lambda}^{2}-8+4 b^{2} \bar{\lambda}^{2}-4 b^{2}$. $\Phi$ is real if $D>0$, so that if $\bar{\lambda}^{2}>1$ then $D$ always is positive. But if $\bar{\lambda}^{2}<1$, then we have the condition that

$$
b^{2} \geq-2+\frac{2}{\bar{\lambda}^{2}}+\frac{2}{\bar{\lambda}^{2}} \sqrt{1-\bar{\lambda}^{4}}
$$

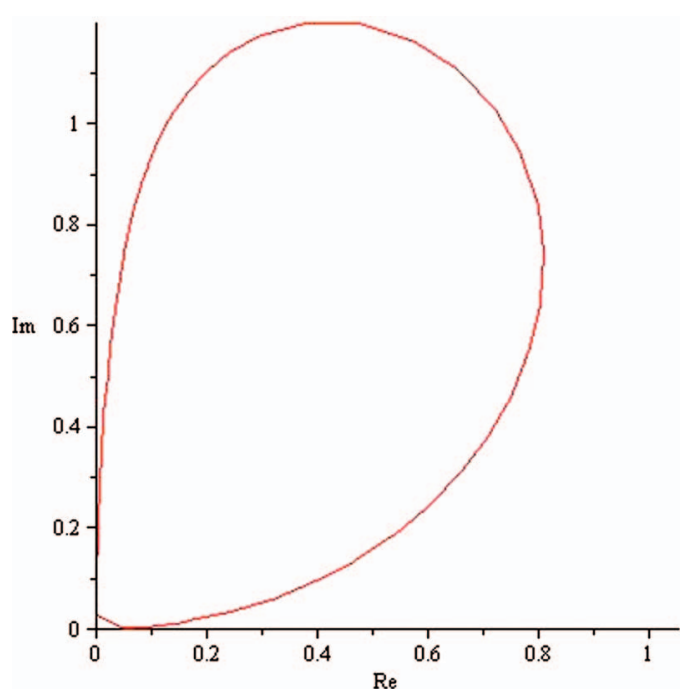

FIG. 20. $\bar{\lambda}=\frac{1}{2}, b=0 ; \mu_{2}$-eigenvalue of $p_{4}$; Plot for $-3 \leq a<0$ 
$\Phi$ is positive if $-\bar{\lambda} b^{3}+b \sqrt{D}>0$. Then, there are two cases: If $b<0$, then $\bar{\lambda}^{2}<1$ and $b^{2} \geq-2+\frac{2}{\bar{\lambda}^{2}}+\frac{2}{\lambda^{2}} \sqrt{1-\bar{\lambda}^{4}}$, which means that $b<-3.75$. Let us consider the case when $a=0$ and $\bar{\lambda}=\frac{1}{2}$. The value of $\Phi$ is

$$
\Phi=\frac{1}{2} b\left(\frac{-b^{2}+\sqrt{b^{4}-12 b^{2}-24}}{b^{2}+2}\right) .
$$

The eigenvalues are given by

$$
\mu_{i}=\frac{3\left(J_{2}-\sqrt{b^{4}-12 b^{2}-24} J_{1} \mp J_{3} \sqrt{b^{4}-12 b^{2}-24} \pm J_{4}\right)}{2\left(b^{2}+2\right)\left(-\sqrt{b^{4}-12 b^{2}-24} J_{5}+J_{6}\right)} .
$$

It can be shown that $p_{5}$ is a saddle when $a=0$ and $\bar{\lambda}=\frac{1}{2}$.

In the case $b>0$, we then have $\bar{\lambda}^{2}>1$. Let us consider the case when $a=0$ and $\bar{\lambda}=2$. The value of $\Phi$ is

$$
\Phi=\frac{1}{2} b\left(\frac{-b^{2}+\sqrt{b^{4}+3 b^{2}+6}}{b^{2}+2}\right) .
$$

We define

$$
\begin{gathered}
k_{1}=13 b^{14}-169 b^{2}-332 b^{10}-620 b^{8}-3232 b^{6}-8000 b^{4}-7680 b^{2}-768, \\
k_{2}=-13 b^{12}+91 b^{10}+488 b^{8}+1508 b^{6}+1936 b^{4}+704 b^{2}, \\
k_{3}=589824+2359296 b^{2}+9535488 b^{4}+10665984 b^{6}, \\
k_{4}=-15224832 b^{10}-3186688 b^{8}-11761280 b^{12}-2668608 b 614 \\
k_{5}=+978 b^{28}-32 b^{30}+1508064 b^{16}+1383072 b^{18}+450120 b^{20} \\
k_{6}=+40428 b^{22}-21822 b^{24}-6004 b^{26}
\end{gathered}
$$

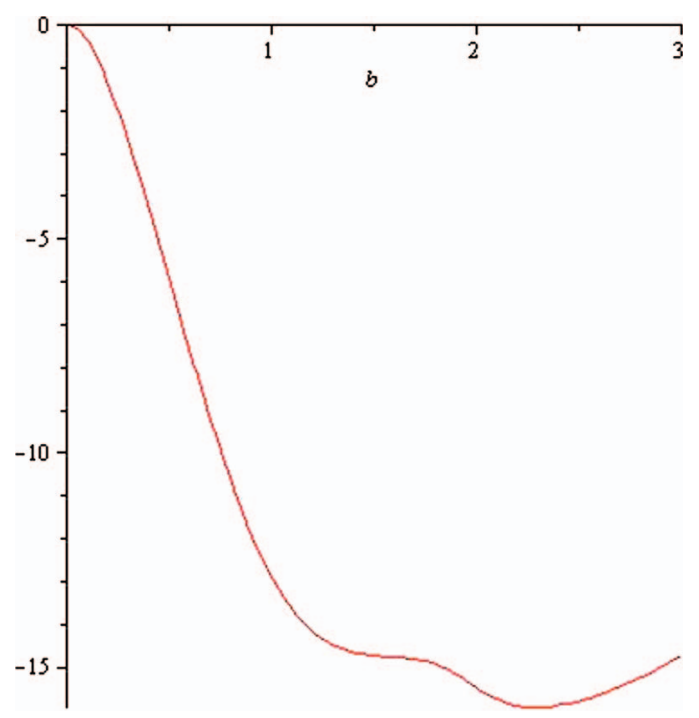

FIG. 21. $\bar{\lambda}=2, a=0$; the real part of $\mu_{1}$-eigenvalue of $p_{5}$; Plot for $0<b \leq 3$. 


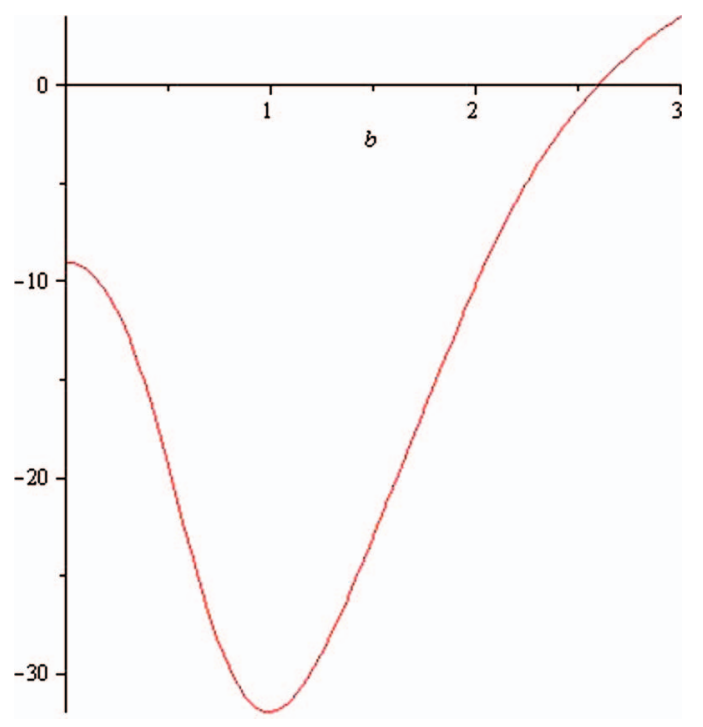

FIG. 22. $\bar{\lambda}=2, a=0$; the real part of $\mu_{2}$-eigenvalue of $p_{5}$; Plot for $0<b \leq 3$.

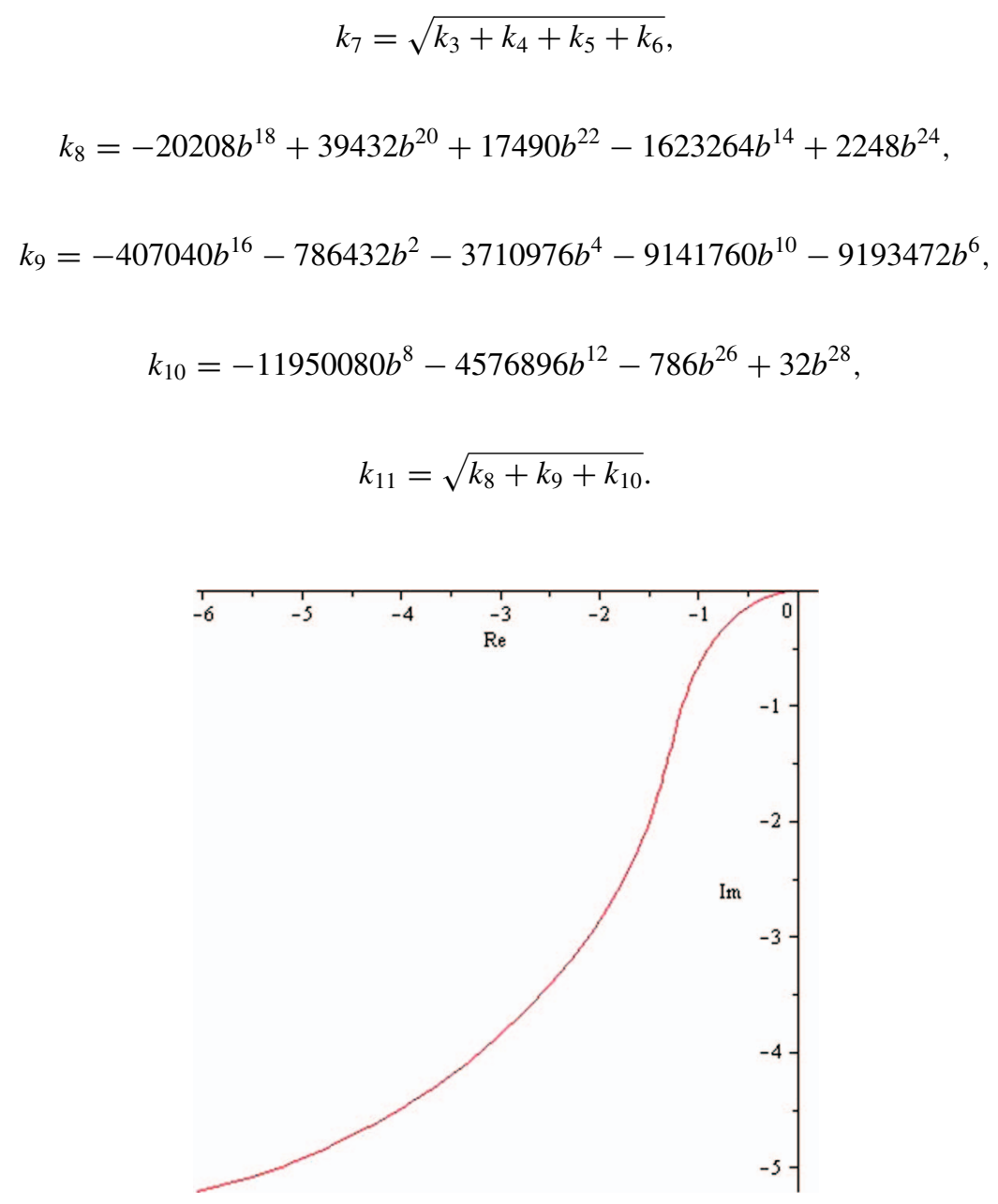

FIG. 23. $\bar{\lambda}=\frac{1}{2}, b=0 ; \mu_{1}$-eigenvalue of $p_{5} ;$ Plot $-2 \leq a<0$. 


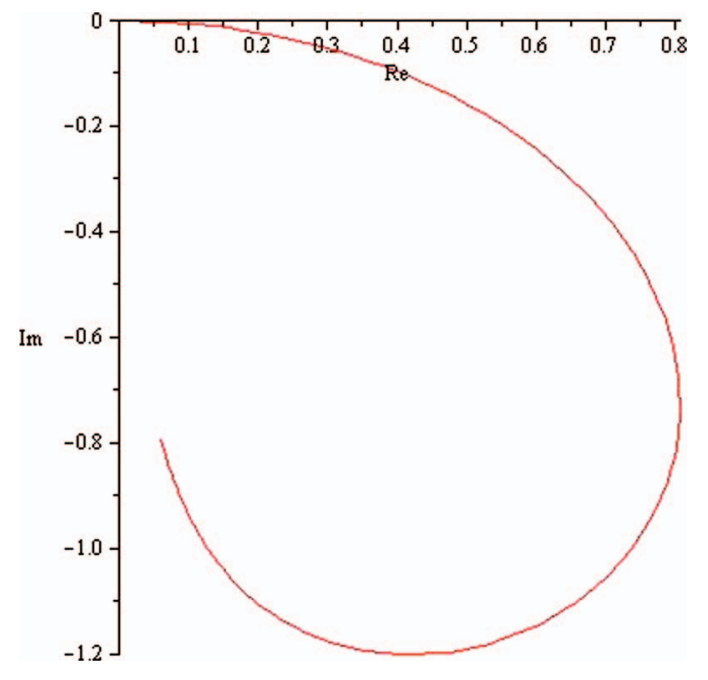

FIG. 24. $\bar{\lambda}=\frac{1}{2}, b=0 ; \mu_{2}$-eigenvalue of $p_{5}$; Plot $-2 \leq a<0$.

The eigenvalues are given by

$$
\mu_{i}=\frac{-3\left(k_{1}+\sqrt{b^{4}-12 b^{2}-24} k_{2} \pm k_{7}+\mp k_{11} \sqrt{b^{4}-12 b^{2}-24}\right)}{2\left(b^{2}+2\right)\left(-\sqrt{b^{4}-12 b^{2}-24} J_{5}+J_{6}\right)} .
$$

We plot the real part of the eigenvalues in Figs. 21 and 22 (where the vertical axis is the real part of the eigenvalues and the horizontal axis is the value of the parameter in the range $0<b \leq 3$ ). As can be seen from Figs. 21 and 22, the real parts of $\mu_{1}$ and $\mu_{2}$ are both negative in the range of $0<b \lesssim 2.55$; therefore, $p_{5}$ is a sink. The real part of $\mu_{2}$ changes sign for $b \simeq 2.55$, so that implies $p_{5}$ becomes a saddle. Note that $q>0$, which implies that $p_{5}$ is not inflationary.

The second case for $p_{5}$ is when $b=0$, and the value of $\Phi$ is

$$
\Phi=-\frac{a}{2}
$$

which is positive if $a<0$. Let us consider the case where $b=0$ and $\bar{\lambda}=\frac{1}{2}$; the eigenvalues are given by

$$
\mu_{i}=\frac{3}{16} a\left(-a^{3}-a n_{2} \pm \overline{n_{6}}\right)
$$

where

$$
n_{2}=\sqrt{\left(a^{2}-6\right)\left(a^{2}+4\right)}, \overline{n_{6}}=\sqrt{2 a^{6}+2 a^{4} n_{2}-20 a^{2}-4 a^{4}+48-2 a^{2} n_{2}} .
$$

We plot the eigenvalues in Figs. 23 and 24 (where the horizontal axis is the real part of the eigenvalues and the vertical axis is the imaginary part of the eigenvalues for the range of the parameter $-2 \leq a<0$ ). As can be seen from these figures, the real part of $\mu_{1}$ is negative in the range $-2 \leq a<0$ but the real part of $\mu_{2}$ is positive is the same range. Thus, $p_{5}$ is a saddle in this case.

\section{B. Summary}

Let us summarize the stability analysis. The point $p_{2}$ is a sink for both cases $(a=0, b=0)$ in the range of values $-3 \leq a, b \leq 2$. However, $p_{3}$ is a saddle when $a=0$ and $\bar{\lambda}=\frac{1}{2}$ for the range $2<b \leq 5$. $p_{3}$ is a source when $b=0$ and $\bar{\lambda}=\sqrt{2}$ in the range $-4 \leq a \leq-1$. The model is inflationary for $p_{2}$ if either $-\frac{1}{2} \lesssim b$ or $a \lesssim 1$. The model is inflationary for $p_{3}$ if either $\frac{1}{2} \lesssim b$ or $a \lesssim-1$. Let us next consider the stability of $p_{4}$. When $a=0$ and $\bar{\lambda}=\frac{1}{2}, p_{4}$ is a source in the range $-7 \leq b \leq-4$. But $p_{4}$ is a saddle when $b=0$ and $\bar{\lambda}=\frac{1}{2}$. For $p_{5}$, there are restrictions on the ranges 
for $a$ and $b$ in order for $p_{5}$ to exist. $p_{5}$ is a sink when $a=0$ and $\bar{\lambda}=2$ in the range of $0<a \lesssim 2.55$, but $q>0$ which implies that $p_{5}$ is not inflationary.

The equilibrium points correspond to physically realistic solutions for a restricted set of values of the parameters. Therefore, a complete discussion of the cosmological models would include further analysis of the viable (physical) ranges for the parameters and the nature of the equilibrium points in each of these viable ranges. However, for each set of parameters in the ranges discussed, there always exists an inflationary future attractor for sufficiently small $\bar{\lambda}$.

\section{CONCLUSIONS}

We have investigated cosmological models in the Einstein-aether theory and (extended) Horava gravity in which both the aether vector field and the metric tensor together determine the evolution. We have been especially interested in the possible inflationary behaviour of the models in a class of spatially anisotropic cosmological models. In particular, we have studied scalar field models in which the self-interaction potential, consisting of terms each containing exponentials, depends on the scalar field $\phi$ and also on the timelike vector field through the expansion rate $\theta$ and the shear scalar $\sigma$. We derived the evolution equations in the anisotropic models, which consist of the energymomentum conservation law or Klein-Gordon equation, the generalized Friedmann equation, the Raychaudhuri equation, and the evolution equations for the shear and for the tilt. It was argued that the tilt generally decays to the future (in the anisotropic models under consideration), and we thus concentrated on the inflationary behaviour of models in the case of negligible tilt.

We introduced expansion-normalized variables and obtained the resulting dimensionless evolution equations which reduce to a 3D dynamical system. We studied the behaviour of the models in general, and in the 2D zero curvature case (which includes the Bianchi I type models) in detail. In particular, we studied the local stability of equilibrium points of the 2D dynamical system corresponding to physically realistic solutions with a restricted set of values of the parameters. We concluded that there are always ranges of values for which there exists an inflationary sink.

In this paper, we have primarily been interested in the local stability of equilibrium points of the cosmological dynamical system and, especially, in demonstrating the local existence of physically important inflationary future attractors (sinks). Some of these local sinks may also be global sinks. We have also briefly discussed the past evolution of the Einstein-aether cosmological models. A global analysis of a particular cosmological model (i.e., a specific Bianchi model with a specific self-interaction potential), based on the dynamical systems approach used here, would certainly be possible. ${ }^{14}$

\section{ACKNOWLEDGMENTS}

We would like to thank William Donnelly, Ted Jacobson, and Genly Leon for helpful comments. This work was supported, in part, by NSERC of Canada. B.A. would also like to thank the Government of Saudi Arabia for financial support.

\section{APPENDIX A: REVIEW OF SCALAR FIELD COSMOLOGY}

Spatially homogeneous and isotropic FRW scalar field models have been studied in Ref. 15. Spatially homogeneous but anisotropic models of various Bianchi types have been studied in Ref. 16 (especially exact solutions) and Ref. 19. A qualitative analysis of all Bianchi models with $k^{2}<2$ (where $k \equiv-\lambda$ ), including standard matter satisfying various energy conditions, was presented in Ref. 17. It was found that the power-law inflationary solution is indeed an attractor for all initially expanding Bianchi models (except for a subclass of the Bianchi type IX models which recollapse). 


\section{Equations}

Cosmological models with a minimally coupled scalar field have an effective energy density and pressure given by

$$
\rho_{\phi}=\frac{1}{2} \dot{\phi}^{2}+V(\phi), p_{\phi}=\frac{1}{2} \dot{\phi}^{2}-V(\phi) .
$$

In the models under consideration, the potential of the scalar field is given by

$$
V(\phi)=V_{0} \mathrm{e}^{k \phi},
$$

where $V_{0}(>0)$ and $k$ are constants.

From the Einstein FE we have the Raychaudhuri equation governing the evolution of the expansion

$$
\dot{\theta}=-2 \sigma^{2}-\frac{1}{3} \theta^{2}-\dot{\phi}^{2}+V(\phi)
$$

and the generalized Friedmann equation

$$
\theta^{2}=3 \sigma^{2}+\frac{3}{2} \dot{\phi}^{2}+3 V(\phi)-\frac{3}{2} P
$$

where $\sigma$ is the shear scalar, $P$ is the scalar curvature of the homogeneous hypersurfaces, which is always negative except in the Bianchi IX case, and $V(\phi)$ is given by Eq. (A2). The Klein-Gordon equation for the scalar field with an exponential potential is then

$$
\ddot{\phi}+\theta \dot{\phi}+k V(\phi)=0 \text {. }
$$

We now introduce new expansion-normalized variables and a new time variable as follows:

$$
\begin{array}{rlrl}
\beta & =\sqrt{3} \frac{\sigma}{\theta}, & & \frac{d t}{d \tau}=\frac{3}{\theta}, \\
\Psi=\frac{\sqrt{6}}{2} \frac{\dot{\phi}}{\theta}, & \Phi & =\sqrt{3 V_{0}} \frac{\mathrm{e}^{k \phi / 2}}{\theta} .
\end{array}
$$

With these definitions, Eqs. (A3)-(A5) can be rewritten as

$$
\begin{aligned}
& \Psi^{\prime}=-\Psi\left(2-2 \beta^{2}-2 \Psi^{2}+\Phi^{2}\right)-\frac{\sqrt{6} k}{2} \Phi^{2}, \\
& \Phi^{\prime}=-\Phi\left(-1-2 \beta^{2}-2 \Psi^{2}+\Phi^{2}-\frac{\sqrt{6} k}{2} \Psi\right),
\end{aligned}
$$

where' denotes differentiation with respect the new time $\tau$. There is an additional differential equation for $\beta$, which depends on the particular Bianchi model under consideration. The equilibrium points of the system have either $\Phi=\Psi=0$, which corresponds to the massless scalar field case, $\beta^{2}$ $+\Psi^{2}=1, \Phi=0$, which represents the Bianchi type I initial (line) singularity, or else (and in all cases of interest here) obey the following relation:

$$
\Phi^{2}+\Psi^{2}=-\frac{\sqrt{6}}{k} \Psi
$$

\section{A class of anisotropic cosmological models}

The diagonal form of the Bianchi type $\mathrm{VI}_{h}$ metric is given by ${ }^{21}$

$$
d s^{2}=-d t^{2}+a(t)^{2} d x^{2}+b(t)^{2} e^{2 m x} d y^{2}+c(t)^{2} e^{2 x} d z^{2}
$$

where $m=h-1$. If $m=1$, then the metric is of Bianchi type $\mathrm{V}$, if $m=0$, then the metric is of Bianchi type III, and if $m=-1$, then the metric is of Bianchi type $\mathrm{VI}_{o}$. Thus, we are considering a 
1-parameter $(m)$ class of Bianchi models which include Bianchi types III $(m=0), \mathrm{V}(m=1), \mathrm{VI}_{0}$ $(m=-1)$, and $\mathrm{VI}_{h}$ (all other $\left.m\right)$.

The expansion scalar (A11), which determines the volume behaviour of the fluid, is given by

$$
\theta=\frac{\dot{a}}{a}+\frac{\dot{b}}{b}+\frac{\dot{c}}{c}
$$

(where an over-dot denotes differentiation with respect to the proper time). The shear tensor, $\sigma_{a b}$, determines the distortion arising in the fluid flow leaving the volume invariant. The nonzero components of the shear tensor are

$$
\begin{aligned}
\sigma_{11} & =\frac{a^{2}}{3}\left(2 \frac{\dot{a}}{a}-\frac{\dot{b}}{b}-\frac{\dot{c}}{c}\right), \\
\sigma_{22} & =\frac{b^{2} \mathrm{e}^{2 m x}}{3}\left(2 \frac{\dot{b}}{b}-\frac{\dot{a}}{a}-\frac{\dot{c}}{c}\right), \\
\sigma_{33} & =\frac{c^{2} \mathrm{e}^{2 x}}{3}\left(2 \frac{\dot{c}}{c}-\frac{\dot{a}}{a}-\frac{\dot{b}}{b}\right),
\end{aligned}
$$

and the shear scalar, $\sigma^{2} \equiv \frac{1}{2} \sigma^{a b} \sigma_{a b}$, is given by

$$
\sigma^{2}=\frac{1}{3}\left[\left(\frac{\dot{a}}{a}\right)^{2}+\left(\frac{\dot{b}}{b}\right)^{2}+\left(\frac{\dot{c}}{c}\right)^{2}-\frac{\dot{a} \dot{b}}{a b}-\frac{\dot{a} \dot{c}}{a c}-\frac{\dot{b} \dot{c}}{b c}\right] .
$$

In the case under consideration here, there is no rotation and no acceleration.

For a scalar field with an exponential potential, the Einstein FE can be written down explicitly. ${ }^{21}$ We obtain the generalized Friedmann equation

$$
\theta^{2}=3 \sigma^{2}+\frac{3}{2} \dot{\phi}^{2}+3 V_{0} \mathrm{e}^{k \phi}+\frac{3}{a^{2}}\left(m^{2}+m+1\right) .
$$

Note that $m^{2}+m+1 \geq 3 / 4>0$. The Raychaudhuri equation (Eq. (A3)) is

$$
\dot{\theta}=-2 \sigma^{2}-\frac{1}{3} \theta^{2}-\dot{\phi}^{2}+V_{0} \mathrm{e}^{k \phi} .
$$

The evolution equation for the shear is

$$
\dot{\sigma}=-\sigma \theta+\frac{(1-m)}{3 \sqrt{3} \sqrt{m^{2}+m+1}}\left(\theta^{2}-3 \sigma^{2}-\frac{3}{2} \dot{\phi}^{2}-3 V_{0} \mathrm{e}^{k \phi}\right) .
$$

The Klein-Gordon equation for the scalar field (Eq. (A5)) is

$$
\ddot{\phi}=-\theta \dot{\phi}-k V_{0} \mathrm{e}^{k \phi} \text {. }
$$

The system of differential equations in the expansion-normalized variables becomes

$$
\begin{aligned}
& \frac{d \beta}{d \tau}=\beta(q-2)+\frac{1-m}{\sqrt{m^{2}+m+1}}\left(1-\beta^{2}-\Psi^{2}-\Phi^{2}\right), \\
& \frac{d \Psi}{d \tau}=\Psi(q-2)-\frac{\sqrt{6} k}{2} \Phi^{2}, \\
& \frac{d \Phi}{d \tau}=\Phi(1+q)+\frac{\sqrt{6} k}{2} \Psi \Phi,
\end{aligned}
$$

and the decoupled evolution equation for the expansion

$$
\frac{d \theta}{d \tau}=-\theta(1+q)
$$

where the deceleration parameter, $q$, is defined by

$$
q=2 \beta^{2}+2 \Psi^{2}-\Phi^{2} .
$$


The domain of interest (determined by Eq. (A14)) is the region defined by

$$
\beta^{2}+\Psi^{2}+\Phi^{2} \leq 1,
$$

which describes the surface and interior of a sphere in the (reduced) phase space $(\beta, \Psi, \Phi)$. Inflation in the context of this paper is defined to occur whenever the deceleration parameter is negative; i.e., $q<0$.

In this brief review we have not included ordinary matter (satisfying the usual energy conditions). However, matter can be easily included (see Ref. 14).

\section{Equilibrium points and qualitative behaviour}

The equilibrium point

$$
\left\{\beta=0, \Psi=-k / \sqrt{6}, \Phi=\sqrt{1-k^{2} / 6}\right\},
$$

does not exist if $k^{2}>6$ and is part of the non-isolated line of equilibrium points $\beta^{2}+\Psi^{2}=1$ when $k^{2}=6$. The point lies on the boundary of the phase space $\beta^{2}+\Psi^{2}+\Phi^{2}=1$ and hence it corresponds to a model with zero curvature. The point is inflationary if $q=\left(k^{2}-2\right) / 2<0$; i.e., if $k^{2}<2$. The eigenvalues are: $\lambda_{1}=\lambda_{2}=\left(k^{2}-6\right) / 2, \lambda_{3}=k^{2}-2$. If $k^{2}<2$ the point is therefore a sink, and if $2<k^{2}<6$, then the point is a saddle point. For $k \neq 0$ the exact solution corresponding to this equilibrium point is that of a flat FRW model. Note: $k^{2} \leq 2$ is a necessary condition for the homogeneous models under consideration to isotropize, ${ }^{17}$ and for $k^{2}<2$ these models will inflate; the power-law inflationary FRW solution is the unique attractor for any initially expanding Bianchi model.

The equilibrium point

$$
\left\{\beta=(1-m) /\left(2 \sqrt{m^{2}+m+1}\right), \Psi=0, \Phi=0\right\},
$$

satisfies the boundary condition, Eq. (A23), for all $m$, and when $m=-1$ the point is part of the non-isolated line of equilibrium points $\beta^{2}+\Psi^{2}=1$. The inflationary condition $q<0$ is never satisfied and hence this point is non-inflationary. The eigenvalues are: $\lambda_{1}=\lambda_{2}=-\lambda_{3}=-3(\mathrm{~m}$ $+1)^{2} /\left(2\left(m^{2}+m+1\right)\right)$. It is easily seen that this point is a saddle point with a two-dimensional stable manifold. The exact solution corresponding to this point is that of a vacuum Bianchi type $\mathrm{VI}_{h}$ model or one of its degeneracies.

There is also an equilibrium point with all of $\beta, \Psi, \Phi$ non-zero when $k^{2} \geq 2$, and the corresponding solution is non-inflationary when the point exists inside the physical phase space given by Eq. (A23). It can be shown that if $k^{2}>2$, all three eigenvalues are negative and hence the equilibrium point is a stable attractor. It is also interesting to note that if $k^{2}>2+4\left(\mathrm{~m}^{2}+1\right) /\left(7 \mathrm{~m}^{2}\right.$ $-2 m+7)$, then the equilibrium point is a focus. The exact solution corresponding to this point is that of a Bianchi type $\mathrm{VI}_{h}$ model or one of its degeneracies. Thus for $k^{2}>2$, this non-isotropic and non-inflationary solution is a stable attractor for the type III and $\mathrm{VI}_{h}$ cases. When $m=1$, the corresponding isotropic solution represents the future asymptotic attractor for the Bianchi type $\mathrm{VII}_{h}$ models as well as the asymptotic attractor for the Bianchi type V models.

In the full three-dimensional phase space the ring of equilibrium points $\left(\beta^{2}+\Psi^{2}=1, \Phi=0\right)$ for $k^{2}<6$ is a global source, and for $k^{2}>6$ we find that some part of the ring acts like a source and the remaining part of the ring acts like a saddle. The equilibrium points corresponding to sources (part of the past attractor) consist of a subset of the Jacobs Disc, which correspond to exact self-similar Jacobs stiff perfect fluid (or massless scalar field) solutions. ${ }^{14}$ Therefore, the models are asymptotic in the past to an analogue of the (non-oscillatory) Jacobs anisotropic Bianchi I non-vacuum solution.

\section{APPENDIX B: GENERAL MODEL}

Let us consider the scalar potential

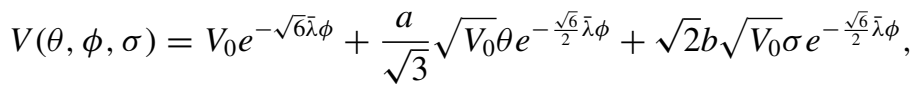


where we have defined $\bar{\lambda}=\lambda / \sqrt{6}$, and we have normalized the constants $a$ and $b$ appropriately. We could also discuss the more general potential $V(\theta, \phi, \sigma)=V_{0} \exp [-\lambda \phi]+a_{r, s} \theta^{r} \sigma^{s} \exp [(r+s$ $-2) \lambda \phi / 2]$.

In general, we have that

$$
\rho_{\phi}=\frac{1}{2} \dot{\phi}^{2}+V-V_{\theta}\left(\theta+\sqrt{6} B_{1} \sigma\right)-V_{\sigma}\left(B_{2} \sigma-\frac{1}{\sqrt{6}} \theta\right),
$$

and an appropriate expression for $p_{\phi}$ in terms of the arbitrary parameters $B_{1}$ and $B_{2}$ (where $B_{1}=1$ and $B_{2}=1$ for the anisotropic models above). We then obtain

$$
\begin{aligned}
\beta^{\prime} & =(q-2) \beta-\frac{\sqrt{2} M}{c^{2}} K, \\
\Psi^{\prime} & =(q-2) \Psi+\frac{3 \bar{\lambda}}{2}\left(2 \Phi^{2}+a \Phi+b \beta \Phi\right), \\
\Phi^{\prime} & =(q+1) \Phi-3 \bar{\lambda} \Psi \Phi,
\end{aligned}
$$

where

$$
\begin{gathered}
K=\frac{c^{2}}{2} \beta^{2}+\Omega-1, \\
\Omega \equiv \Psi^{2}+\Phi^{2}-a B_{1} \Phi \beta-b\left(B_{2}-1\right) \Phi \beta+b \Phi, \\
q-2=\frac{K}{b \Phi-2}\left[\left(a B_{1}+\left(B_{2}-1\right) b\right) \frac{\sqrt{2} M}{c^{2}} \Phi-4\right]+ \\
\frac{\Phi}{b \Phi-2}\left[3(\bar{\lambda} \Psi-1)\left(a B_{1}+b B_{2}\right) \beta+3(a-b) \bar{\lambda} \Psi+3 b(\beta+1)+6 \Phi\right],
\end{gathered}
$$

The system (B3)-(B5), with arbitrary parameters (including $B_{1}$ and $B_{2}$ ), can be regarded as a phenomenological system of equations for an anisotropic cosmological model with a particular self-interaction potential $V(\theta, \phi, \sigma)$.

We note that when $K=0$, from (B3) the equilibrium points must satisfy $\beta=0$ or $q=2$. In the latter case we have $\Phi=0$ and $\Psi^{2}+\frac{c^{2}}{2} \beta^{2}=1$, which will include the cosmological sources. When $\beta=0$ we will find equilibrium points (with $\Phi=0$ and $\Psi$ non-zero) corresponding to inflationary sinks.

\section{2D system with zero curvature}

From Eqs. (B3)-(B5), we can compute the evolution equation for the auxiliary variable $K$; by setting $K=0$ in this expression we obtain $K^{\prime}=0$, so that $K=0$ is an invariant set of the system. We recall that we can obtain the Bianchi I models (with $K=0$ ) when $M=N=0$. When $K=0$ we obtain

$$
\begin{aligned}
& \beta^{\prime}=(q-2) \beta, \\
& \Phi^{\prime}=(q+1) \Phi-3 \bar{\lambda} \Psi \Phi,
\end{aligned}
$$

where

$$
\begin{aligned}
\Psi^{2} & =1-\frac{c^{2}}{2} \beta^{2}-\Phi^{2}+\left(B_{2}-1\right) b \beta \Phi+a B_{1} \beta \Phi-b \Phi, \\
q-2 & =\frac{3 \Phi}{b \Phi-2}\left[(\bar{\lambda} \Psi-1)\left(a B_{1}+b B_{2}\right) \beta+(a-b) \bar{\lambda} \Psi+b(\beta+1)+2 \Phi\right] .
\end{aligned}
$$


${ }^{1}$ W. Donnelly and T. Jacobson, Phys. Rev. D 82, 064032 (2010).

${ }^{2}$ T. Jacobson and D. Mattingly, Phys. Rev. D 64, 024028 (2001).

${ }^{3}$ P. Horava, Phys. Rev. D 79, 084008 (2009); T. Jacobson, ibid. 81, 101502 (2010).

${ }^{4}$ D. Wiltshire, P. Smale, T. Mattsson, and R. Watkins, e-print arXiv:1201.5371 [astro-ph.CO].

${ }^{5}$ K. A. Olive, Phys. Rep. 190, 307 (1990); A. D. Linde, "Inflation and quantum cosmology," in 300 Years of Gravitation, edited by S. W. Hawking and W. Israel (Cambridge University Press, Cambridge, 1987), pp. 604-630.

${ }^{6}$ I. Carruthers and T. Jacobson, Phys. Rev. D 83, 024034 (2011), e-print arXiv:1011.6466 [gr-qc].

${ }^{7}$ S. Kanno and J. Soda, Phys. Rev. D 74, 063505 (2006).

${ }^{8}$ T. G. Zlosnik, P. G. Ferreira, and G. D. Starkman, Phys. Rev. D 75, 044017 (2007).

${ }^{9}$ J. D. Barrow, Phys. Rev. D 85, 047503 (2012), e-print arXiv:1201.2882.

${ }^{10}$ M. Gasparini, Phys. Lett. B 163, 84 (1985); B. Li, D. F. Mota, and J. D. Barrow, Phys. Rev. D 77, 024032 (2008); J. A. Zuntz, P. G. Ferreira, and T. G. Zlosnik, Phys. Rev. Lett. 101, 261102 (2008); T. Clifton, P. G. Ferreira, A. Padilla, and C. Skordis, Phys. Rep. 513, 1 (2012), e-print arXiv:1106.2476 [astro-ph.CO].

${ }^{11}$ E. Cremmer, S. Ferrara, C. Kounnas, and D. V. Nanopoulos, Phys. Lett. B 133, 61 (1983); J. Ellis, A. B. Lahanas, D. V. Nanopoulos, and K. Tamvakis, ibid. 134, 429 (1984); A. Salam and E. Sezgin, ibid. 147, 47 (1984); K. Maeda and H. Nishino, ibid. 154, 358 (1985); 158, 381 (1985); L. J. Romans, Nucl. Phys. B 269, 691 (1986).

${ }^{12}$ A. R. Liddle, A. Mazumdar, and F. E. Schunck, Phys. Rev. D 58, 061301 (1998); A. A. Coley and R. J. van den Hoogen, ibid. 62, 023517 (2000), e-print arXiv:gr-qc/9911075; K. A. Malik and D. Wands, ibid. 59, 123501 (1999); E. J. Copeland, A. Mazumdar, and N. J. Nunes, ibid. 60, 083506 (1999); A. M. Green and J. E. Lidsey, ibid. 61, 067301 (2000).

${ }^{13}$ A. P. Billyard, A. A. Coley, R. J. van den Hoogen, J. Ibañez, and I. Olasagasti, Class. Quantum Grav. 16, 4035 (1999), e-print arXiv:gr-qc/9907053; E. J. Copeland, A. R. Liddle, and D. Wands, Phys. Rev. D 57, 4686 (1998); R. J. van den Hoogen, A. A. Coley, and D. Wands, Class. Quantum Grav. 16, 1843 (1999), e-print arXiv:gr-qc/9901014; A. P. Billyard, A. A. Coley, and R. J. van den Hoogen, Phys. Rev. D 58, 123501 (1998).

${ }^{14}$ A. A. Coley, Dynamical Systems and Cosmology (Kluwer Academic, Dordrecht, 2003).

${ }^{15}$ J. J. Halliwell, Phys. Lett. B 185, 341 (1987).

${ }^{16}$ A. B. Burd, and J. D. Barrow, Nucl. Phys. B 308, 929 (1988); J. Lidsey, Class. Quantum Grav. 9, 1239 (1992); A. Feinstein and J. Ibáñez, ibid. 10, 93 (1993); J. M. Aguirrebiria, A. Feinstein, and J. Ibáñez, Phys. Rev. D 48, 4662 (1993).

${ }^{17}$ Y. Kitada and K. Maeda, Phys. Rev. D 45, 1416 (1992); Class. Quantum Grav. 10, 703 (1993).

${ }^{18}$ J. Khoury, B. A. Ovrut, P. J. Steinhardt, and N. Turok, Phys. Rev. D 64, 123522 (2001), e-print arXiv:hep-th/0103239; A. Coley and W. C. Lim, Class. Quantum Grav. 22, 3073 (2005), e-print arXiv:gr-qc/0506097.

${ }^{19}$ J. Ibáñez, R. J. van den Hoogen, and A. A. Coley, Phys. Rev. D 51, 928 (1995); R. J. van den Hoogen, A. A. Coley, and J. Ibáñez, ibid. 55, 5215 (1997).

${ }^{20}$ P. Sandin, B. Alhulaimi, and A. Coley, Phys. Rev. D 87, 044031 (2013), e-print arXiv:1211.4402.

${ }^{21}$ A. A. Coley, J. Ibáñez, and R. J. van den Hoogen, J. Math. Phys. 38, 5256 (1997).

${ }^{22}$ A. A. Coley and S. Hervik, Class. Quantum Grav. 22, 579 (2005); S. Hervik, R. J. van den Hoogen, and A. A. Coley, ibid. 22, 607 (2005); S. Hervik, R. J. van den Hoogen, W. C. Lim, and A. A. Coley, ibid. 23, 845 (2006); A. A. Coley, S. Hervik, and W. C. Lim, ibid. 23, 3573 (2006).

${ }^{23}$ T. Jacobson, Status Report No. PoSQG-Ph:020, 2007; e-print arXiv:0801.1547 [gr-qc]. 